CRYSTALLOGRAPHIC COMMUNICATIONS

ISSN 2056-9890

Received 15 July 2019

Accepted 9 August 2019

Edited by K. Fejfarova, Institute of Biotechnology CAS, Czech Republic

Keywords: crystal structure; polypyrazolyl borate; iron(II) scorpionates; magnetism; C$\mathrm{H} \cdots \pi$ interactions.

CCDC reference: 1946393

Supporting information: this article has supporting information at journals.iucr.org/e

\section{Crystal structure and magnetic properties of bis[butyltris(1H-pyrazol-1-yl)borato]iron(II)}

\author{
Maksym Seredyuk, ${ }^{\mathrm{a} *}$ Kateryna Znovjyak, $^{\mathrm{a}, \mathrm{b}}$ Igor O. Fritsky, ${ }^{\mathrm{a}, \mathrm{b}}$ Tatiana Y. Sliva $^{\mathrm{a}}$ and \\ Mykola S. Slobodyanik ${ }^{\mathrm{a}}$ \\ a Department of Chemistry, Taras Shevchenko National University of Kyiv, Volodymyrska Street 64, Kyiv, 01601, Ukraine, \\ and ${ }^{\mathbf{b}}$ UkrOrgSyntez Ltd, Chervonotkatska Street 67, Kyiv 02094, Ukraine. *Correspondence e-mail: mcs@univ.kiev.ua
}

The asymmetric unit of the title compound, $\left[\mathrm{Fe}\left(\mathrm{C}_{13} \mathrm{H}_{18} \mathrm{BN}_{6}\right)_{2}\right]$, contains two half independent complex molecules. In each complex, the $\mathrm{Fe}^{\mathrm{II}}$ atom is located on an inversion center and is surrounded by two scorpionate ligand butyltris $(1 \mathrm{H}$ pyrazol-1-yl)borate molecules that coordinate to the iron(II) ion through the $\mathrm{N}$ atoms of the pyrazole groups. The two independent complex molecules differ essentially in the conformation of the butyl substituents. In the crystal, the complex molecules are linked by a series of $\mathrm{C}-\mathrm{H} \cdots \pi$ interactions, which generate a supramolecular three-dimensional structure. At $120 \mathrm{~K}$, the average $\mathrm{Fe}-\mathrm{N}$ bond distance is $1.969 \AA$, indicating the low-spin state of the iron(II) atom, which does not change upon heating, as demonstrated by hightemperature magnetic susceptibility measurements.

\section{Chemical context}

Scorpionates, coordination metal complexes of poly(1pyrazolyl)borates, have been studied intensively since the pioneering work of Trofimenko (1999). Iron(II) derivatives are particularly interesting because of the spin-state crossover between ${ }^{1} A_{1}$ low-spin (LS) and ${ }^{5} T_{2 \mathrm{~g}}$ high-spin (HS) observed for several scorpionate ligands (Long et al., 2004; Halcrow, 2007). Complexes of this type are sensitive to the effects induced by substituents on the electronic structure of the ligand and/or steric crowding (Hamon et al., 2008). The prototypical $\left[\mathrm{Fe}\left(\mathrm{HB}(\mathrm{pz})_{3}\right)_{2}\right]$, the LS compound at $295 \mathrm{~K}$, undergoes a spin-state crossover to the HS state upon heating to $c a 420 \mathrm{~K}$ (Long et al., 2004). Introducing methyl substituents to the pyrazole moieties decreases the ligand field and shifts the spin crossover down in temperature or completely stabilizes the high-spin state of the iron(II) ion (Long et al., 2004). In contrast, scorpionate ligands bearing an organic substituent instead of the hydrogen atom on the hub boron atom demonstrate stabilization of the low-spin state and shift of the spin transition to the higher temperature range (Hamon et al., 2008).

Our continuing interest consists of a study of iron(II) complexes bearing alkyl chains (Seredyuk, 2012; Seredyuk et al., 2006, 2010, 2016) and those based on azol ligands (Seredyuk et al., 2007, 2015). Here we report on the synthesis, crystal structure and magnetic properties of an alkylated charge-neutral iron(II) complex based on the scorpionate ligand butyltris( $1 H$-pyrazol-1-yl)borate. 


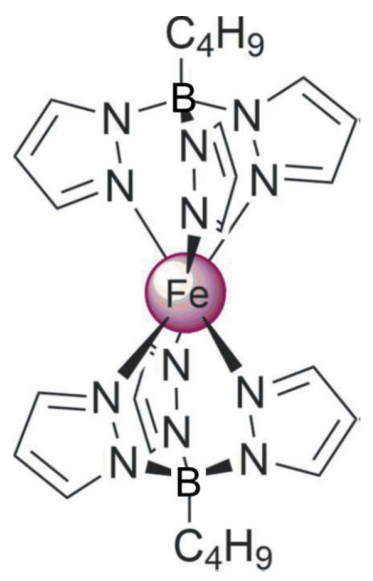

\section{Structural commentary}

The asymmetric unit of the title compound contains two half independent complex molecules. In each complex, the $\mathrm{Fe}^{\mathrm{II}}$ atom, that is located on an inversion center, is surrounded by two scorpionate ligands; each one providing three pyrazole moieties coordinated in a fac mode, thus a pseudo-octahedral $\left[\mathrm{FeN}_{6}\right]$ coordination polyhedron is formed (Fig. 1). The two complex molecules differ essentially in the conformation of the butyl groups. One of the methylene groups of the butyl substituents of the Fe1-based complex shows a gauche conformation, whilst the remaining two methylene groups are
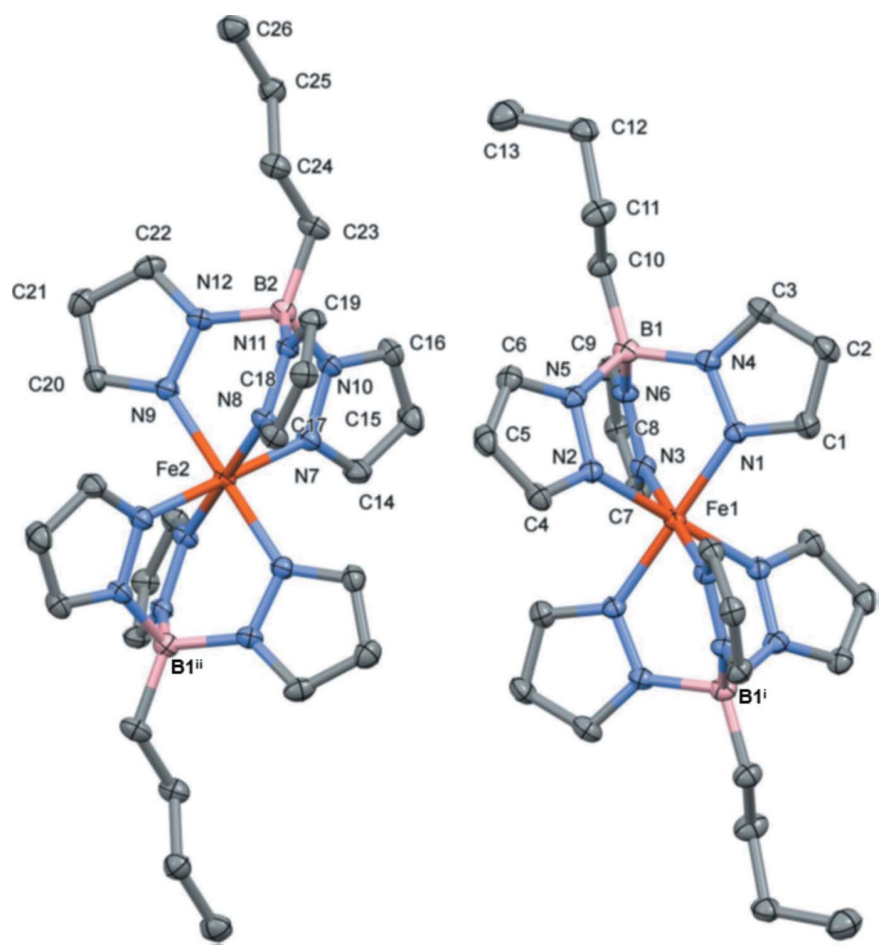

Figure 1

Molecular structure of the two complex molecules of the title compound showing the atom labelling [symmetry codes: (i) $-x,-y,-z+1$; (ii) $-x+1,-y,-z+1]$. Displacement ellipsoids are drawn at the $50 \%$ probability level. For clarity, $\mathrm{H}$ atoms have been omitted.
Table 1

Selected bond lengths $(\AA)$.

\begin{tabular}{llll}
\hline $\mathrm{Fe} 1-\mathrm{N} 1$ & $1.956(2)$ & $\mathrm{Fe} 2-\mathrm{N} 9$ & $1.963(2)$ \\
$\mathrm{Fe} 1-\mathrm{N} 2$ & $1.969(3)$ & $\mathrm{Fe} 2-\mathrm{N} 7$ & $1.977(3)$ \\
$\mathrm{Fe} 1-\mathrm{N} 3$ & $1.971(2)$ & $\mathrm{Fe} 2-\mathrm{N} 8$ & $1.977(2)$ \\
\hline
\end{tabular}

Table 2

Hydrogen-bond geometry $\left(\AA,^{\circ}\right)$.

$C g 2$ and $C g 11$ are the centroids of rings N2/N5/C4-C6 and N8/N11/C17-C19, respectively.

\begin{tabular}{lllll}
\hline$D-\mathrm{H} \cdots A$ & $D-\mathrm{H}$ & $\mathrm{H} \cdots A$ & $D \cdots A$ & $D-\mathrm{H} \cdots A$ \\
\hline $\mathrm{C} 5-\mathrm{H} 5 \cdots C g 11$ & 0.93 & 3.00 & $3.674(4)$ & 131 \\
$\mathrm{C} 12-\mathrm{H} 12 A \cdots C g 2^{\mathrm{i}}$ & 0.97 & 2.87 & $3.703(3)$ & 145 \\
$\mathrm{C} 26-\mathrm{H} 26 C \cdots C g 11^{\mathrm{ii}}$ & 0.96 & 2.84 & $3.720(4)$ & 153 \\
\hline
\end{tabular}

Symmetry codes: (i) $-x, y+\frac{1}{2},-z+\frac{1}{2}$; (ii) $-x+1, y+\frac{1}{2},-z+\frac{1}{2}$.

in the trans conformation. Oppositely, the three methylene groups of the butyl substituent of the Fe2-based molecule are close to a trans conformation (Fig. 1).

The average $\mathrm{Fe}-\mathrm{N}$ bond length is $1.969 \AA$ (Table 1), a typical value for the low-spin state of the iron(II) ion (Gütlich \& Goodwin, 2004). The average trigonal distortion parameters $\Phi=\Sigma_{1}{ }^{24}\left(60-\theta_{\mathrm{i}}\right) / 24$, where $\theta_{\mathrm{i}}$ is the angle generated by superposition of two opposite faces of the octahedron (Chang et al. 1990,) and $\Sigma=\Sigma_{1}{ }^{12}\left(\left|\phi_{\mathrm{i}}-90\right|\right)$, where $\phi_{\mathrm{i}}$ are the deviations from $90^{\circ}$ of the $c i s-\mathrm{N}-\mathrm{Fe}-\mathrm{N}$ angles in the coordination sphere (Drew et al. 1995), are 1.27 and $24.38^{\circ}$, respectively, which correspond to a relatively low distortion of the coordination polyhedron and are typical for the low-spin state of iron(II) (Guionneau et al., 2004). The averaged volume of the coordination polyhedron is equal to $10.155 \AA^{3}$.

\section{Supramolecular features}

In the crystal, molecules are linked by $\mathrm{C}-\mathrm{H} \cdots \pi$ interactions (Fig. 2, Table 2). The pyrazole-butyl or butyl-butyl contacts

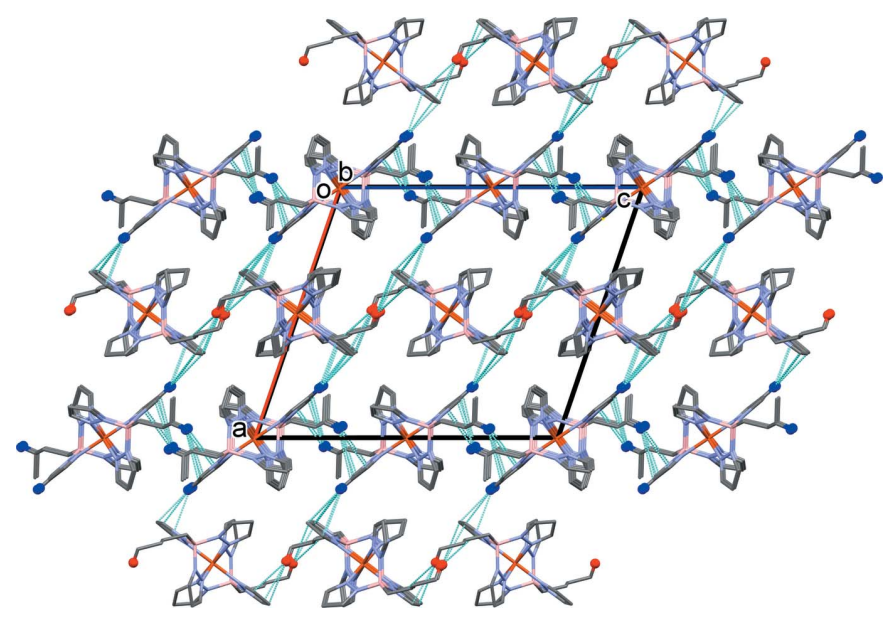

Figure 2

A view along the $b$ axis of the crystal packing of the title compound, with the $\mathrm{C}-\mathrm{H} \cdots \pi$ contacts (see Table 2 for details) represented by dashed lines. For clarity, only the $\mathrm{H}$ atoms involved are shown, as blue balls for the Fe1 complex molecule and red balls for the Fe2 complex molecule. 


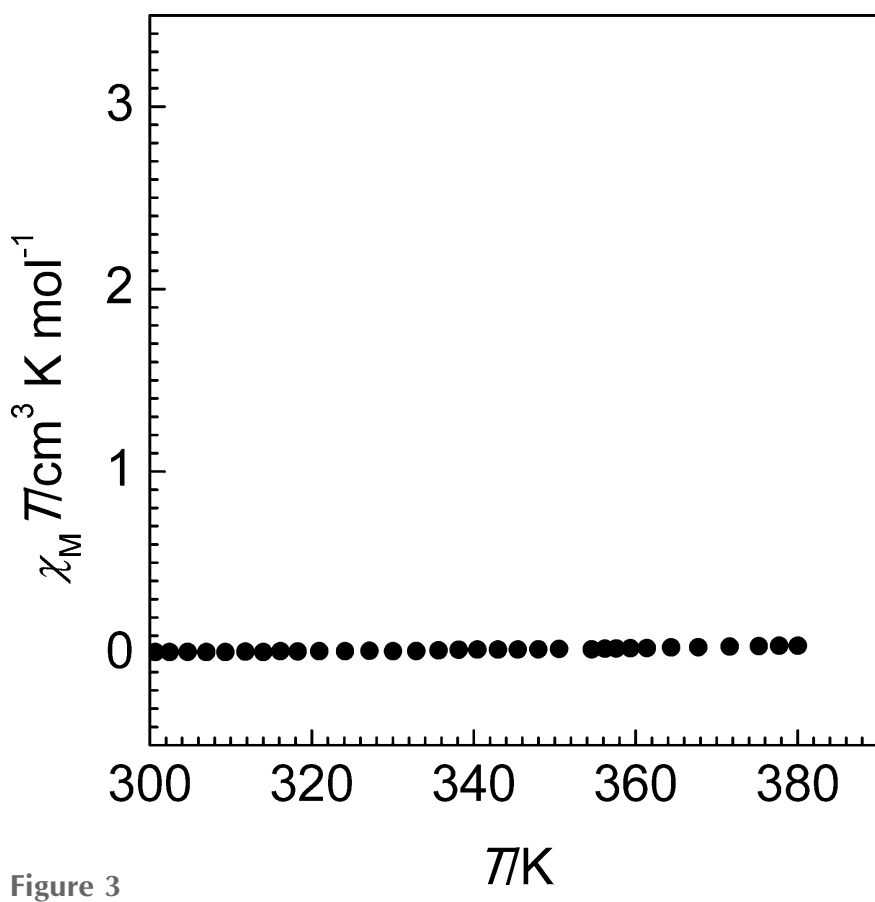

$\chi_{\mathrm{M}} T$ versus $T$ plot for the title compound.

link the individual complex molecules to form layers parallel to the $b c$ plane. The layers are linked by $\mathrm{C}-\mathrm{H} \cdots \pi$ pyrazolepyrazole interactions (Table 2), leading to the formation of a supramolecular three-dimensional structure, as shown in Fig. 2.

\section{Magnetic measurements}

Variable-temperature magnetic susceptibility measurements were performed on single crystals $(20 \mathrm{mg})$ of the title compound using a Quantum Design MPMS2 superconducting quantum interference device (SQUID) susceptometer operating at $1 \mathrm{~T}$ in the temperature range $10-400 \mathrm{~K}$. Experimental susceptibilities were corrected for the diamagnetism of the holder (gelatine capsule) and of the constituent atoms by the application of Pascal's constants. The magnetic behaviour of the compound recorded at $2 \mathrm{~K} \mathrm{~min}^{-1}$ is shown in Fig. 3 in the form of $\chi_{\mathrm{M}} T$ versus $T\left(\chi_{\mathrm{M}}\right.$ is the molar magnetic susceptibility and $T$ is the temperature). At $300 \mathrm{~K}$, the $\chi_{\mathrm{M}} T$ value is close to zero, and on heating the value remains constant up to $400 \mathrm{~K}$. This corroborates well with the observed short average $\mathrm{Fe}-\mathrm{N}$ bond length at $120 \mathrm{~K}$ and identifies the low-spin state of the central iron(II) ion.

\section{Database survey}

A search of the Cambridge Structural Database (CSD, Version 5.39, update November 2017; Groom et al., 2016) for complexes containing the iron(II) ion based on a scorpionate ligand with a tri( $1 H$-pyrazol-1-yl)borate fragment yielded 39 hits, with $\mathrm{Fe}-\mathrm{N}$ bond lengths lying in the ranges 1.956-1.995 and $2.162-2.246 \AA$, respectively, for the low- and high-spin states of the iron(II) ion.
Table 3

Experimental details.

\begin{tabular}{|c|c|}
\hline \multicolumn{2}{|l|}{ Crystal data } \\
\hline Chemical formula & {$\left[\mathrm{Fe}\left(\mathrm{C}_{13} \mathrm{H}_{18} \mathrm{BN}_{6}\right)_{2}\right]$} \\
\hline$M_{\mathrm{r}}$ & 594.14 \\
\hline Crystal system, space group & Monoclinic, $P 2_{1} / c$ \\
\hline Temperature $(\mathrm{K})$ & 120 \\
\hline$a, b, c(\AA)$ & $\begin{array}{l}16.0762(4), 10.1827(2) \\
\quad 18.3410(3)\end{array}$ \\
\hline$\beta\left(^{\circ}\right)$ & $108.785(2)$ \\
\hline$V\left(\AA^{3}\right)$ & $2842.48(11)$ \\
\hline$Z$ & 4 \\
\hline Radiation type & Мо $K \alpha$ \\
\hline$\mu\left(\mathrm{mm}^{-1}\right)$ & 0.57 \\
\hline Crystal size (mm) & $0.08 \times 0.04 \times 0.04$ \\
\hline \multicolumn{2}{|l|}{ Data collection } \\
\hline Diffractometer & Agilent SuperNova Sapphire3 \\
\hline Absorption correction & $\begin{array}{l}\text { Multi-scan (CrysAlis PRO; } \\
\text { Agilent, 2012) }\end{array}$ \\
\hline$T_{\min }, T_{\max }$ & $0.769,1.000$ \\
\hline $\begin{array}{l}\text { No. of measured, independent and } \\
\text { observed }[I>2 \sigma(I)] \text { reflections }\end{array}$ & $17726,7222,4596$ \\
\hline$R_{\text {int }}$ & 0.053 \\
\hline$(\sin \theta / \lambda)_{\max }\left(\AA^{-1}\right)$ & 0.700 \\
\hline \multicolumn{2}{|l|}{ Refinement } \\
\hline$R\left[F^{2}>2 \sigma\left(F^{2}\right)\right], w R\left(F^{2}\right), S$ & $0.053,0.184,0.78$ \\
\hline No. of reflections & 7222 \\
\hline No. of parameters & 373 \\
\hline $\mathrm{H}$-atom treatment & H-atom parameters constrained \\
\hline$\Delta \rho_{\max }, \Delta \rho_{\min }\left(\mathrm{e} \AA^{-3}\right)$ & $0.39,-0.71$ \\
\hline
\end{tabular}

Computer programs: CrysAlis PRO (Agilent, 2012), SHELXT2014 (Sheldrick, 2015a), Mercury (Macrae et al., 2008), SHELXL2014 (Sheldrick, 2015b), PLATON (Spek, 2009) and publCIF (Westrip, 2010).

\section{Synthesis and crystallization}

The butyltris( $1 H$-pyrazol-1-yl)borate ligand and the title compound were synthesized according to the reported procedures (Reger \& Tarquini, 1982; Myers et al., 2008). The slow diffusion of hexane vapour into a chloroform solution of the title compound led to the separation of orange wellshaped crystals.

Elemental analysis for $\mathrm{C}_{26} \mathrm{H}_{36} \mathrm{~B}_{2} \mathrm{FeN}_{12}$ (found): $\mathrm{C}, 52.56 ; \mathrm{H}$, $6.11 ; \mathrm{N}, 28.29 \%$; (calculated): C, 52.22; H, 6.05; N, 28.38\%.

\section{Refinement}

Crystal data, data collection and structure refinement details are summarized in Table 3 . The $\mathrm{H}$-atoms were included in calculated positions and treated as riding atoms: $\mathrm{C}-\mathrm{H}=0.93-$ $0.97 \AA$ 我 with $U_{\text {iso }}(\mathrm{H})=1.2 U_{\text {eq }}(\mathrm{C})$.

\section{Funding information}

Funding for this research was provided by: H2020 Marie Skłodowska-Curie Actions (grant No. 734322).

\section{References}

Agilent (2012). CrysAlis PRO. Agilent Technologies Ltd, Yarnton, England.

Chang, H. R., McCusker, J. K., Toftlund, H., Wilson, S. R., Trautwein, A. X., Winkler, H. \& Hendrickson, D. N. (1990). J. Am. Chem. Soc. 112, 6814-6827. 
Drew, M. G. B., Harding, C. J., McKee, V., Morgan, G. G. \& Nelson, J. (1995). J. Chem. Soc. Chem. Commun. pp. 1035-1038.

Groom, C. R., Bruno, I. J., Lightfoot, M. P. \& Ward, S. C. (2016). Acta Cryst. B72, 171-179.

Guionneau, P., Marchivie, M., Bravic, G., Létard, J. F. \& Chasseau, D. (2004). Top. Curr. Chem. 234, 97-128.

Gütlich, P. \& Goodwin, H. A. (2004). Top. Curr. Chem. 233, 1-47.

Halcrow, M. A. (2007). Polyhedron, 26, 3523-3576.

Hamon, P., Thépot, J. Y., Le Floch, M., Boulon, M. E., Cador, O., Golhen, S., Ouahab, L., Fadel, L., Saillard, J. Y. \& Hamon, J. R. (2008). Angew. Chem. Int. Ed. 47, 8687-8691.

Long, G. J., Grandjean, F. \& Reger, D. L. (2004). Top. Curr. Chem. 233, 91-122.

Macrae, C. F., Bruno, I. J., Chisholm, J. A., Edgington, P. R., McCabe, P., Pidcock, E., Rodriguez-Monge, L., Taylor, R., van de Streek, J. \& Wood, P. A. (2008). J. Appl. Cryst. 41, 466-470.

Myers, W. K., Duesler, E. N. \& Tierney, D. L. (2008). Inorg. Chem. 47, 6701-6710.

Reger, D. L. \& Tarquini, M. E. (1982). Inorg. Chem. 21, 840-842.

Seredyuk, M. (2012). Inorg. Chim. Acta, 380, 65-71.
Seredyuk, M., Gaspar, A. B., Ksenofontov, V., Galyametdinov, Y., Verdaguer, M., Villain, F. \& Gütlich, P. (2010). Inorg. Chem. 49, 10022-10031.

Seredyuk, M., Gaspar, A. B., Ksenofontov, V., Reiman, S., Galyametdinov, Y., Haase, W., Rentschler, E. \& Gütlich, P. (2006). Hyperfine Interact. 166, 385-390.

Seredyuk, M., Haukka, M., Fritsky, I. O., Kozłowski, H., Krämer, R., Pavlenko, V. A. \& Gütlich, P. (2007). Dalton Trans. pp. 31833194.

Seredyuk, M., Piñeiro-López, L., Muñoz, M. C., Martínez-Casado, F. J., Molnár, G., Rodriguez-Velamazán, J. A., Bousseksou, A. \& Real, J. A. (2015). Inorg. Chem. 54, 7424-7432.

Seredyuk, M., Znovjyak, K., Muñoz, M. C., Galyametdinov, Y., Fritsky, I. O. \& Real, J. A. (2016). RSC Adv. 6, 39627-39635.

Sheldrick, G. M. (2015a). Acta Cryst. A71, 3-8.

Sheldrick, G. M. (2015b). Acta Cryst. C71, 3-8.

Spek, A. L. (2009). Acta Cryst. D65, 148-155.

Trofimenko, S. (1999). Scorpionates - the Coordination Chemistry of Polypyrazylborate Ligands. London: Imperial College Press.

Westrip, S. P. (2010). J. Appl. Cryst. 43, 920-925. 


\section{supporting information}

Acta Cryst. (2019). E75, 1327-1330 [https://doi.org/10.1107/S2056989019011137]

\section{Crystal structure and magnetic properties of bis[butyltris $(1 \mathrm{H}$-pyrazol-1-}

\section{yl)borato]iron(II)}

\section{Maksym Seredyuk, Kateryna Znovjyak, Igor O. Fritsky, Tatiana Y. Sliva and Mykola S.}

\section{Slobodyanik}

\section{Computing details}

Data collection: CrysAlis PRO (Agilent, 2012); cell refinement: CrysAlis PRO (Agilent, 2012); data reduction: CrysAlis PRO (Agilent, 2012); program(s) used to solve structure: SHELXT2014 (Sheldrick, 2015a); program(s) used to refine structure: SHELXL2014 (Sheldrick, 2015b); molecular graphics: Mercury (Macrae et al., 2008); software used to prepare material for publication: SHELXL2014 (Sheldrick, 2015b), PLATON (Spek, 2009) and publCIF (Westrip, 2010).

Bis[butyltris(1H-pyrazol-1-yl)borato]iron(II)

Crystal data

$\left[\mathrm{Fe}\left(\mathrm{C}_{13} \mathrm{H}_{18} \mathrm{BN}_{6}\right)_{2}\right]$

$M_{r}=594.14$

Monoclinic, $P 2{ }_{1} / c$

$a=16.0762(4) \AA$

$b=10.1827(2) \AA$

$c=18.3410(3) \AA$

$\beta=108.785(2)^{\circ}$

$V=2842.48(11) \AA^{3}$

$Z=4$

\section{Data collection}

Agilent SuperNova Sapphire3 diffractometer

$\varphi$ scans and $\omega$ scans with $\kappa$ offset

Absorption correction: multi-scan

(CrysAlis PRO; Agilent, 2012)

$T_{\text {min }}=0.769, T_{\max }=1.000$

17726 measured reflections

\section{Refinement}

Refinement on $F^{2}$

Least-squares matrix: full

$R\left[F^{2}>2 \sigma\left(F^{2}\right)\right]=0.053$

$w R\left(F^{2}\right)=0.184$

$S=0.78$

7222 reflections

373 parameters

0 restraints
$F(000)=1248$

$D_{\mathrm{x}}=1.388 \mathrm{Mg} \mathrm{m}^{-3}$

Mo $K \alpha$ radiation, $\lambda=0.71073 \AA$

Cell parameters from 2385 reflections

$\theta=3.9-24.7^{\circ}$

$\mu=0.57 \mathrm{~mm}^{-1}$

$T=120 \mathrm{~K}$

Prismatic, orange

$0.08 \times 0.04 \times 0.04 \mathrm{~mm}$

7222 independent reflections

4596 reflections with $I>2 \sigma(I)$

$R_{\text {int }}=0.053$

$\theta_{\text {max }}=29.8^{\circ}, \theta_{\min }=2.9^{\circ}$

$h=-21 \rightarrow 21$

$k=-12 \rightarrow 14$

$l=-23 \rightarrow 24$

Primary atom site location: dual

Secondary atom site location: difference Fourier map

Hydrogen site location: inferred from neighbouring sites

$\mathrm{H}$-atom parameters constrained

$w=1 /\left[\sigma^{2}\left(F_{\mathrm{o}}^{2}\right)+(0.1086 P)^{2}+7.1302 P\right]$

where $P=\left(F_{\mathrm{o}}{ }^{2}+2 F_{\mathrm{c}}{ }^{2}\right) / 3$ 
$(\Delta / \sigma)_{\max }=0.042$

$\Delta \rho_{\min }=-0.71$ e $\AA^{-3}$

$\Delta \rho_{\max }=0.39 \mathrm{e} \AA^{-3}$

Special details

Experimental. CrysAlisPro, Agilent Technologies, Version 1.171.36.21 (release 14-08-2012 CrysAlis171 .NET) (compiled Sep 14 2012,17:21:16) Empirical absorption correction using spherical harmonics, implemented in SCALE3 ABSPACK scaling algorithm.

Geometry. Bond distances, angles etc. have been calculated using the rounded fractional coordinates. All su's are estimated from the variances of the (full) variance-covariance matrix. The cell esds are taken into account in the estimation of distances, angles and torsion angles

Fractional atomic coordinates and isotropic or equivalent isotropic displacement parameters $\left(\AA^{2}\right)$

\begin{tabular}{|c|c|c|c|c|}
\hline & $x$ & $y$ & $z$ & $U_{\text {iso }} * / U_{\text {eq }}$ \\
\hline $\mathrm{Fe} 1$ & 0.00000 & 0.00000 & 0.50000 & $0.0155(2)$ \\
\hline $\mathrm{Fe} 2$ & 0.50000 & 0.00000 & 0.50000 & $0.0164(2)$ \\
\hline N1 & $-0.08358(16)$ & $0.1221(2)$ & $0.43205(13)$ & $0.0180(7)$ \\
\hline $\mathrm{N} 2$ & $0.07144(17)$ & $0.0187(2)$ & $0.43129(14)$ & $0.0190(7)$ \\
\hline N3 & $0.06259(17)$ & $0.1526(2)$ & $0.55863(13)$ & $0.0186(7)$ \\
\hline N4 & $-0.05505(17)$ & $0.2339(2)$ & $0.40633(13)$ & $0.0191(7)$ \\
\hline N5 & $0.09021(17)$ & $0.1412(3)$ & 0.40957 (14) & $0.0195(7)$ \\
\hline N6 & $0.07608(17)$ & $0.2625(2)$ & $0.52172(13)$ & $0.0185(7)$ \\
\hline $\mathrm{C} 1$ & $-0.1716(2)$ & 0.1239 & $0.40482(17)$ & $0.0233(9)$ \\
\hline $\mathrm{C} 2$ & $-0.2013(2)$ & 0.2375 & $0.36109(18)$ & $0.0256(9)$ \\
\hline $\mathrm{C} 3$ & -0.1259 & $0.3041(3)$ & $0.36344(17)$ & $0.0238(9)$ \\
\hline $\mathrm{C} 4$ & $0.1166(2)$ & $-0.0672(3)$ & $0.40370(17)$ & $0.0232(9)$ \\
\hline C5 & $0.1659(2)$ & $-0.0017(3)$ & $0.36471(18)$ & $0.0257(10)$ \\
\hline C6 & $0.1480(2)$ & $0.1291(3)$ & $0.37018(17)$ & $0.0219(9)$ \\
\hline $\mathrm{C} 7$ & $0.0940(2)$ & $0.1777(3)$ & $0.63425(16)$ & $0.0211(8)$ \\
\hline $\mathrm{C} 8$ & $0.1281(2)$ & $0.3052(3)$ & $0.64677(17)$ & $0.0212(8)$ \\
\hline C9 & $0.1151(2)$ & $0.3554(3)$ & $0.57409(17)$ & $0.0215(8)$ \\
\hline $\mathrm{C} 10$ & $0.0694(2)$ & $0.3998(3)$ & 0.40007 (17) & $0.0229(9)$ \\
\hline $\mathrm{C} 11$ & $0.0458(2)$ & 0.4211 & $0.31252(17)$ & $0.0281(10)$ \\
\hline $\mathrm{C} 12$ & $0.0626(2)$ & $0.5604(3)$ & $0.28979(18)$ & $0.0263(9)$ \\
\hline $\mathrm{C} 13$ & $0.1591(3)$ & $0.5980(4)$ & $0.3142(2)$ & $0.0374(11)$ \\
\hline B1 & $0.0458(2)$ & 0.2628 & $0.43210(18)$ & $0.0196(9)$ \\
\hline N7 & $0.42396(17)$ & $0.1237(3)$ & $0.53253(14)$ & $0.0206(7)$ \\
\hline N8 & $0.42220(17)$ & $0.0242(2)$ & $0.39276(14)$ & $0.0191(7)$ \\
\hline N9 & $0.56735(17)$ & $0.1520(2)$ & $0.48428(14)$ & $0.0199(7)$ \\
\hline N10 & $0.39344(17)$ & $0.2352(2)$ & $0.49125(14)$ & $0.0200(7)$ \\
\hline N11 & $0.39382(16)$ & $0.1463(2)$ & $0.36572(14)$ & $0.0175(7)$ \\
\hline N12 & $0.52540(17)$ & $0.2641(2)$ & $0.45014(14)$ & $0.0195(7)$ \\
\hline $\mathrm{C} 14$ & $0.3881(2)$ & 0.1201 & $0.58890(18)$ & $0.0254(9)$ \\
\hline $\mathrm{C} 15$ & $0.3332(2)$ & $0.2279(4)$ & $0.58413(19)$ & $0.0292(10)$ \\
\hline $\mathrm{C} 16$ & $0.3382(2)$ & 0.2988 & $0.52117(17)$ & $0.0229(9)$ \\
\hline $\mathrm{C} 17$ & $0.3881(2)$ & -0.0589 & $0.33497(17)$ & $0.0222(9)$ \\
\hline $\mathrm{C} 18$ & $0.3368(2)$ & 0.0083 & $0.26958(18)$ & $0.0234(9)$ \\
\hline C19 & $0.3418(2)$ & $0.1382(3)$ & $0.29165(17)$ & $0.0214(8)$ \\
\hline $\mathrm{C} 20$ & $0.6534(2)$ & $0.1765(3)$ & $0.50184(17)$ & $0.0228(9)$ \\
\hline
\end{tabular}




\begin{tabular}{|c|c|c|c|c|}
\hline $\mathrm{C} 21$ & $0.6682(2)$ & $0.3032(3)$ & $0.48037(18)$ & $0.0262(9)$ \\
\hline $\mathrm{C} 22$ & $0.5852(2)$ & $0.3555(3)$ & $0.44766(17)$ & $0.0241(9)$ \\
\hline $\mathrm{C} 23$ & $0.3840(2)$ & $0.4040(3)$ & $0.38150(18)$ & $0.0245(9)$ \\
\hline $\mathrm{C} 24$ & $0.4056(2)$ & $0.4516(3)$ & $0.30977(17)$ & $0.0240(9)$ \\
\hline $\mathrm{C} 25$ & $0.4168(2)$ & $0.6000(3)$ & $0.30874(18)$ & $0.0244(9)$ \\
\hline $\mathrm{C} 26$ & $0.4399(2)$ & $0.6506(3)$ & $0.2394(2)$ & $0.0299(10)$ \\
\hline B2 & $0.4229(2)$ & $0.2666(3)$ & $0.42016(19)$ & $0.0194(9)$ \\
\hline $\mathrm{H} 1$ & -0.20780 & 0.05890 & 0.41370 & $0.0280 *$ \\
\hline $\mathrm{H} 2$ & -0.25920 & 0.26240 & 0.33600 & $0.0310^{*}$ \\
\hline H3 & -0.12370 & 0.38400 & 0.33960 & $0.0280 *$ \\
\hline $\mathrm{H} 4$ & 0.11540 & -0.15790 & 0.40960 & $0.0280^{*}$ \\
\hline H5 & 0.20280 & -0.03860 & 0.34030 & $0.0310^{*}$ \\
\hline H6 & 0.17170 & 0.19810 & 0.35010 & $0.0260^{*}$ \\
\hline $\mathrm{H} 7$ & 0.09340 & 0.11890 & 0.67290 & $0.0250^{*}$ \\
\hline H8 & 0.15380 & 0.34700 & 0.69380 & $0.0250 *$ \\
\hline H9 & 0.13070 & 0.43920 & 0.56300 & $0.0260 *$ \\
\hline $\mathrm{H} 10 \mathrm{~A}$ & 0.04100 & 0.46890 & 0.41980 & $0.0280 *$ \\
\hline H10B & 0.13220 & 0.41290 & 0.42270 & $0.0280 *$ \\
\hline H11A & 0.07970 & 0.36020 & 0.29270 & $0.0340 *$ \\
\hline H11B & -0.01580 & 0.40030 & 0.28820 & $0.0340 *$ \\
\hline $\mathrm{H} 12 \mathrm{~A}$ & 0.03790 & 0.56890 & 0.23430 & $0.0320 *$ \\
\hline $\mathrm{H} 12 \mathrm{~B}$ & 0.03210 & 0.62170 & 0.31270 & $0.0320^{*}$ \\
\hline $\mathrm{H} 13 \mathrm{~A}$ & 0.16460 & 0.68650 & 0.29830 & $0.0450 *$ \\
\hline H13B & 0.18960 & 0.53960 & 0.29040 & $0.0450 *$ \\
\hline $\mathrm{H} 13 \mathrm{C}$ & 0.18400 & 0.59160 & 0.36920 & $0.0450 *$ \\
\hline $\mathrm{H} 14$ & 0.39830 & 0.05470 & 0.62610 & $0.0310^{*}$ \\
\hline $\mathrm{H} 15$ & 0.30040 & 0.24800 & 0.61620 & $0.0350 *$ \\
\hline H16 & 0.30870 & 0.37670 & 0.50270 & $0.0270 *$ \\
\hline H17 & 0.39710 & -0.14930 & 0.33770 & $0.0270 *$ \\
\hline H18 & 0.30590 & -0.02690 & 0.22170 & $0.0280^{*}$ \\
\hline H19 & 0.31410 & 0.20820 & 0.26080 & $0.0260 *$ \\
\hline $\mathrm{H} 20$ & 0.69760 & 0.11660 & 0.52530 & $0.0270 *$ \\
\hline $\mathrm{H} 21$ & 0.72190 & 0.34360 & 0.48660 & $0.0310^{*}$ \\
\hline $\mathrm{H} 22$ & 0.57260 & 0.43960 & 0.42730 & $0.0290 *$ \\
\hline $\mathrm{H} 23 \mathrm{~A}$ & 0.32050 & 0.39940 & 0.36780 & $0.0290 *$ \\
\hline $\mathrm{H} 23 \mathrm{~B}$ & 0.40370 & 0.47130 & 0.42070 & $0.0290 *$ \\
\hline $\mathrm{H} 24 \mathrm{~A}$ & 0.45930 & 0.40960 & 0.30860 & $0.0290 *$ \\
\hline $\mathrm{H} 24 \mathrm{~B}$ & 0.35870 & 0.42520 & 0.26390 & $0.0290 *$ \\
\hline $\mathrm{H} 25 \mathrm{~A}$ & 0.36270 & 0.64140 & 0.30930 & $0.0290 *$ \\
\hline $\mathrm{H} 25 \mathrm{~B}$ & 0.46280 & 0.62610 & 0.35530 & $0.0290 *$ \\
\hline $\mathrm{H} 26 \mathrm{~A}$ & 0.44570 & 0.74440 & 0.24240 & $0.0360 *$ \\
\hline $\mathrm{H} 26 \mathrm{~B}$ & 0.39420 & 0.62690 & 0.19290 & $0.0360^{*}$ \\
\hline $\mathrm{H} 26 \mathrm{C}$ & 0.49440 & 0.61230 & 0.23920 & $0.0360 *$ \\
\hline
\end{tabular}

Atomic displacement parameters $\left(\AA^{2}\right)$

\begin{tabular}{lllllll}
\hline & $U^{11}$ & $U^{22}$ & $U^{33}$ & $U^{12}$ & $U^{13}$ & $U^{23}$ \\
\hline $\mathrm{Fe} 1$ & $0.0161(3)$ & $0.0176(3)$ & $0.0129(3)$ & $0.0013(2)$ & $0.0048(2)$ & $0.0009(2)$
\end{tabular}




\begin{tabular}{|c|c|c|c|c|c|c|}
\hline $\mathrm{Fe} 2$ & $0.0173(3)$ & $0.0149(3)$ & $0.0166(3)$ & $0.0033(2)$ & $0.0051(2)$ & $0.0013(2)$ \\
\hline N1 & $0.0194(12)$ & $0.0201(12)$ & $0.0148(11)$ & $0.0013(10)$ & $0.0061(9)$ & $0.0020(10)$ \\
\hline $\mathrm{N} 2$ & $0.0184(12)$ & $0.0223(13)$ & $0.0163(11)$ & $0.0013(10)$ & $0.0057(9)$ & $0.0019(10)$ \\
\hline N3 & $0.0209(12)$ & $0.0192(12)$ & $0.0156(11)$ & $0.0009(10)$ & $0.0059(9)$ & $0.0000(10)$ \\
\hline N4 & $0.0213(13)$ & $0.0182(12)$ & $0.0168(11)$ & $0.0016(10)$ & $0.0049(10)$ & $0.0020(10)$ \\
\hline N5 & $0.0219(13)$ & $0.0216(13)$ & $0.0152(11)$ & $-0.0002(10)$ & $0.0064(9)$ & $0.0022(10)$ \\
\hline N6 & $0.0225(13)$ & $0.0176(12)$ & $0.0148(11)$ & $0.0008(10)$ & $0.0052(9)$ & $0.0008(10)$ \\
\hline $\mathrm{C} 1$ & $0.0200(15)$ & $0.0247(16)$ & $0.0244(15)$ & $0.0014(12)$ & $0.0062(12)$ & $0.0010(13)$ \\
\hline $\mathrm{C} 2$ & $0.0200(15)$ & $0.0265(17)$ & $0.0261(15)$ & $0.0064(13)$ & $0.0017(12)$ & $0.0035(14)$ \\
\hline $\mathrm{C} 3$ & $0.0256(16)$ & $0.0216(16)$ & $0.0203(14)$ & $0.0063(13)$ & $0.0021(12)$ & $0.0014(12)$ \\
\hline $\mathrm{C} 4$ & $0.0229(16)$ & $0.0256(16)$ & $0.0209(14)$ & $0.0070(13)$ & $0.0068(12)$ & $-0.0007(13)$ \\
\hline $\mathrm{C} 5$ & $0.0232(16)$ & $0.0352(19)$ & $0.0218(15)$ & $0.0076(14)$ & $0.0114(12)$ & $0.0010(14)$ \\
\hline C6 & $0.0217(15)$ & $0.0277(17)$ & $0.0168(13)$ & $-0.0007(13)$ & $0.0068(11)$ & $0.0007(12)$ \\
\hline $\mathrm{C} 7$ & $0.0214(15)$ & $0.0262(16)$ & $0.0153(13)$ & $0.0045(12)$ & $0.0052(11)$ & $0.0005(12)$ \\
\hline $\mathrm{C} 8$ & $0.0205(15)$ & $0.0241(16)$ & $0.0181(13)$ & $0.0010(12)$ & $0.0050(11)$ & $-0.0052(12)$ \\
\hline C9 & $0.0221(15)$ & $0.0223(15)$ & $0.0201(14)$ & $-0.0033(12)$ & $0.0069(12)$ & $-0.0012(12)$ \\
\hline $\mathrm{C} 10$ & $0.0250(16)$ & $0.0249(16)$ & $0.0184(14)$ & $-0.0029(13)$ & $0.0063(12)$ & $0.0003(13)$ \\
\hline $\mathrm{C} 11$ & $0.0348(18)$ & $0.0306(18)$ & $0.0174(14)$ & $-0.0065(15)$ & $0.0065(13)$ & $-0.0004(13)$ \\
\hline $\mathrm{C} 12$ & $0.0299(17)$ & $0.0262(17)$ & $0.0230(15)$ & $0.0048(14)$ & $0.0088(13)$ & $0.0075(14)$ \\
\hline $\mathrm{C} 13$ & $0.040(2)$ & $0.039(2)$ & $0.0321(18)$ & $-0.0056(17)$ & $0.0101(16)$ & $0.0066(17)$ \\
\hline B1 & $0.0233(17)$ & $0.0221(17)$ & $0.0139(14)$ & $0.0010(14)$ & $0.0067(12)$ & $0.0001(13)$ \\
\hline N7 & $0.0216(13)$ & 0.0199 (13) & $0.0198(12)$ & $0.0046(10)$ & $0.0058(10)$ & $0.0027(10)$ \\
\hline $\mathrm{N} 8$ & $0.0209(13)$ & $0.0164(12)$ & $0.0191(12)$ & $0.0039(10)$ & $0.0051(10)$ & $0.0000(10)$ \\
\hline N9 & $0.0206(13)$ & $0.0176(12)$ & $0.0207(12)$ & $0.0013(10)$ & $0.0055(10)$ & $0.0020(10)$ \\
\hline N10 & $0.0203(12)$ & $0.0174(12)$ & $0.0219(12)$ & $0.0050(10)$ & $0.0063(10)$ & $0.0011(10)$ \\
\hline N11 & $0.0191(12)$ & $0.0160(12)$ & $0.0177(11)$ & $0.0035(10)$ & $0.0065(9)$ & $0.0020(10)$ \\
\hline N12 & $0.0226(13)$ & $0.0154(12)$ & $0.0199(12)$ & $0.0013(10)$ & $0.0059(10)$ & $0.0014(10)$ \\
\hline $\mathrm{C} 14$ & $0.0303(17)$ & $0.0257(16)$ & $0.0233(15)$ & $0.0061(14)$ & $0.0128(13)$ & $0.0063(13)$ \\
\hline $\mathrm{C} 15$ & $0.0294(18)$ & $0.0345(19)$ & $0.0278(16)$ & $0.0075(15)$ & $0.0151(14)$ & $-0.0001(15)$ \\
\hline $\mathrm{C} 16$ & $0.0212(15)$ & $0.0241(16)$ & $0.0229(14)$ & $0.0057(12)$ & $0.0065(12)$ & $-0.0012(13)$ \\
\hline $\mathrm{C} 17$ & $0.0260(16)$ & $0.0176(15)$ & $0.0223(14)$ & $0.0016(12)$ & $0.0070(12)$ & $-0.0036(12)$ \\
\hline $\mathrm{C} 18$ & $0.0245(16)$ & $0.0224(16)$ & $0.0210(14)$ & $-0.0017(12)$ & $0.0042(12)$ & $-0.0010(12)$ \\
\hline C19 & $0.0236(15)$ & $0.0190(15)$ & $0.0195(14)$ & $0.0009(12)$ & $0.0040(12)$ & $0.0047(12)$ \\
\hline $\mathrm{C} 20$ & $0.0176(14)$ & $0.0288(17)$ & $0.0213(14)$ & $0.0033(12)$ & $0.0052(11)$ & $0.0018(13)$ \\
\hline $\mathrm{C} 21$ & $0.0240(16)$ & $0.0284(17)$ & $0.0259(15)$ & $-0.0044(13)$ & 0.0077 (13) & $-0.0010(14)$ \\
\hline $\mathrm{C} 22$ & $0.0307(17)$ & $0.0195(15)$ & $0.0208(14)$ & $-0.0063(13)$ & $0.0067(12)$ & $-0.0009(12)$ \\
\hline $\mathrm{C} 23$ & $0.0306(17)$ & $0.0177(15)$ & $0.0227(15)$ & $0.0064(13)$ & $0.0051(13)$ & $0.0004(12)$ \\
\hline $\mathrm{C} 24$ & $0.0296(17)$ & $0.0204(15)$ & $0.0206(14)$ & $0.0036(13)$ & $0.0061(12)$ & $0.0012(13)$ \\
\hline $\mathrm{C} 25$ & $0.0272(17)$ & $0.0207(15)$ & $0.0268(15)$ & $0.0010(13)$ & $0.0108(13)$ & $-0.0020(13)$ \\
\hline $\mathrm{C} 26$ & $0.0372(19)$ & $0.0220(16)$ & $0.0335(17)$ & $0.0024(14)$ & $0.0155(15)$ & $-0.0002(14)$ \\
\hline B2 & $0.0199(16)$ & $0.0185(16)$ & $0.0187(15)$ & $0.0007(13)$ & 0.0047 (12) & $-0.0011(13)$ \\
\hline
\end{tabular}

Geometric parameters ( $\left.\AA,{ }^{\circ}\right)$

\begin{tabular}{llll}
\hline $\mathrm{Fe} 1-\mathrm{N} 1$ & $1.956(2)$ & $\mathrm{N} 9-\mathrm{N} 12$ & $1.370(3)$ \\
$\mathrm{Fe} 1-\mathrm{N} 2$ & $1.969(3)$ & $\mathrm{C} 9-\mathrm{H} 9$ & 0.9300 \\
$\mathrm{Fe} 1-\mathrm{N} 3$ & $1.971(2)$ & $\mathrm{N} 9-\mathrm{C} 20$ & $1.339(4)$ \\
$\mathrm{Fe} 1-\mathrm{N} 1 \mathrm{i}$ & $1.956(2)$ & $\mathrm{C} 10-\mathrm{H} 10 \mathrm{~A}$ & 0.9700
\end{tabular}




\begin{tabular}{|c|c|c|c|}
\hline $\mathrm{Fe} 1-\mathrm{N} 2^{\mathrm{i}}$ & $1.969(3)$ & $\mathrm{N} 10-\mathrm{C} 16$ & $1.350(4)$ \\
\hline $\mathrm{Fe} 1-\mathrm{N} 3^{\mathrm{i}}$ & $1.971(2)$ & $\mathrm{N} 10-\mathrm{B} 2$ & 1.557 (4) \\
\hline $\mathrm{Fe} 2-\mathrm{N} 9^{\mathrm{ii}}$ & $1.963(2)$ & $\mathrm{C} 10-\mathrm{H} 10 \mathrm{~B}$ & 0.9700 \\
\hline $\mathrm{Fe} 2-\mathrm{N} 9$ & $1.963(2)$ & N11-C19 & $1.349(4)$ \\
\hline $\mathrm{Fe} 2-\mathrm{N} 7$ & $1.977(3)$ & C11-H11A & 0.9700 \\
\hline $\mathrm{Fe} 2-\mathrm{N} 8$ & $1.977(2)$ & C11-H11B & 0.9700 \\
\hline $\mathrm{Fe} 2-\mathrm{N} 7^{\mathrm{ii}}$ & $1.977(3)$ & $\mathrm{N} 11-\mathrm{B} 2$ & $1.554(4)$ \\
\hline $\mathrm{Fe} 2-\mathrm{N} 8^{\mathrm{ii}}$ & 1.977 (2) & $\mathrm{C} 12-\mathrm{H} 12 \mathrm{~A}$ & 0.9700 \\
\hline $\mathrm{N} 1-\mathrm{C} 1$ & $1.341(4)$ & $\mathrm{C} 12-\mathrm{H} 12 \mathrm{~B}$ & 0.9700 \\
\hline $\mathrm{N} 1-\mathrm{N} 4$ & $1.367(3)$ & $\mathrm{N} 12-\mathrm{C} 22$ & $1.349(4)$ \\
\hline $\mathrm{N} 2-\mathrm{N} 5$ & $1.372(4)$ & $\mathrm{N} 12-\mathrm{B} 2$ & $1.560(4)$ \\
\hline $\mathrm{N} 2-\mathrm{C} 4$ & $1.336(4)$ & $\mathrm{C} 13-\mathrm{H} 13 \mathrm{~A}$ & 0.9600 \\
\hline N3-N6 & $1.361(3)$ & $\mathrm{C} 13-\mathrm{H} 13 \mathrm{~B}$ & 0.9600 \\
\hline $\mathrm{N} 3-\mathrm{C} 7$ & $1.339(4)$ & $\mathrm{C} 13-\mathrm{H} 13 \mathrm{C}$ & 0.9600 \\
\hline $\mathrm{N} 4-\mathrm{B} 1$ & $1.564(4)$ & $\mathrm{C} 14-\mathrm{C} 15$ & $1.394(5)$ \\
\hline $\mathrm{N} 4-\mathrm{C} 3$ & $1.360(4)$ & $\mathrm{C} 15-\mathrm{C} 16$ & $1.386(5)$ \\
\hline N5-C6 & $1.354(4)$ & $\mathrm{C} 17-\mathrm{C} 18$ & $1.396(4)$ \\
\hline N5-B1 & $1.550(5)$ & $\mathrm{C} 18-\mathrm{C} 19$ & $1.378(4)$ \\
\hline N6-C9 & $1.350(4)$ & $\mathrm{C} 20-\mathrm{C} 21$ & $1.392(4)$ \\
\hline N6-B1 & $1.557(4)$ & $\mathrm{C} 21-\mathrm{C} 22$ & $1.382(5)$ \\
\hline $\mathrm{C} 1-\mathrm{C} 2$ & $1.401(4)$ & $\mathrm{C} 23-\mathrm{C} 24$ & $1.543(4)$ \\
\hline $\mathrm{C} 2-\mathrm{C} 3$ & $1.378(5)$ & $\mathrm{C} 23-\mathrm{B} 2$ & $1.602(4)$ \\
\hline $\mathrm{C} 4-\mathrm{C} 5$ & $1.396(5)$ & $\mathrm{C} 24-\mathrm{C} 25$ & $1.523(4)$ \\
\hline $\mathrm{C} 5-\mathrm{C} 6$ & $1.373(4)$ & $\mathrm{C} 25-\mathrm{C} 26$ & $1.525(5)$ \\
\hline $\mathrm{C} 7-\mathrm{C} 8$ & $1.399(4)$ & C14-H14 & 0.9300 \\
\hline $\mathrm{C} 8-\mathrm{C} 9$ & $1.379(4)$ & C15-H15 & 0.9300 \\
\hline $\mathrm{C} 10-\mathrm{C} 11$ & $1.542(4)$ & C16-H16 & 0.9300 \\
\hline $\mathrm{C} 10-\mathrm{B} 1$ & $1.606(5)$ & C17-H17 & 0.9300 \\
\hline $\mathrm{C} 11-\mathrm{C} 12$ & $1.527(4)$ & C18-H18 & 0.9300 \\
\hline $\mathrm{C} 12-\mathrm{C} 13$ & $1.519(6)$ & C19-H19 & 0.9300 \\
\hline $\mathrm{C} 1-\mathrm{H} 1$ & 0.9300 & $\mathrm{C} 20-\mathrm{H} 20$ & 0.9300 \\
\hline $\mathrm{C} 2-\mathrm{H} 2$ & 0.9300 & $\mathrm{C} 21-\mathrm{H} 21$ & 0.9300 \\
\hline $\mathrm{C} 3-\mathrm{H} 3$ & 0.9300 & $\mathrm{C} 22-\mathrm{H} 22$ & 0.9300 \\
\hline $\mathrm{C} 4-\mathrm{H} 4$ & 0.9300 & $\mathrm{C} 23-\mathrm{H} 23 \mathrm{~A}$ & 0.9700 \\
\hline C5-H5 & 0.9300 & $\mathrm{C} 23-\mathrm{H} 23 \mathrm{~B}$ & 0.9700 \\
\hline $\mathrm{C} 6-\mathrm{H} 6$ & 0.9300 & $\mathrm{C} 24-\mathrm{H} 24 \mathrm{~A}$ & 0.9700 \\
\hline N7-N10 & $1.365(4)$ & $\mathrm{C} 24-\mathrm{H} 24 \mathrm{~B}$ & 0.9700 \\
\hline N7-C14 & $1.337(4)$ & $\mathrm{C} 25-\mathrm{H} 25 \mathrm{~A}$ & 0.9700 \\
\hline C7-H7 & 0.9300 & $\mathrm{C} 25-\mathrm{H} 25 \mathrm{~B}$ & 0.9700 \\
\hline $\mathrm{C} 8-\mathrm{H} 8$ & 0.9300 & $\mathrm{C} 26-\mathrm{H} 26 \mathrm{~A}$ & 0.9600 \\
\hline N8-C17 & $1.329(4)$ & $\mathrm{C} 26-\mathrm{H} 26 \mathrm{~B}$ & 0.9600 \\
\hline $\mathrm{N} 8-\mathrm{N} 11$ & $1.362(3)$ & $\mathrm{C} 26-\mathrm{H} 26 \mathrm{C}$ & 0.9600 \\
\hline $\mathrm{N} 1-\mathrm{Fe} 1-\mathrm{N} 2$ & $87.32(10)$ & $\mathrm{N} 6-\mathrm{C} 9-\mathrm{H} 9$ & 126.00 \\
\hline $\mathrm{N} 1-\mathrm{Fe} 1-\mathrm{N} 3$ & $88.38(9)$ & $\mathrm{C} 8-\mathrm{C} 9-\mathrm{H} 9$ & 126.00 \\
\hline $\mathrm{N} 1-\mathrm{Fe} 1-\mathrm{N} 1^{\mathrm{i}}$ & 180.00 & $\mathrm{~N} 12-\mathrm{N} 9-\mathrm{C} 20$ & $105.8(2)$ \\
\hline $\mathrm{N} 1-\mathrm{Fe} 1-\mathrm{N} 2^{\mathrm{i}}$ & $92.68(10)$ & $\mathrm{C} 16-\mathrm{N} 10-\mathrm{B} 2$ & $131.2(2)$ \\
\hline $\mathrm{N} 1-\mathrm{Fe} 1-\mathrm{N} 3^{\mathrm{i}}$ & $91.62(9)$ & $\mathrm{C} 11-\mathrm{C} 10-\mathrm{H} 10 \mathrm{~B}$ & 107.00 \\
\hline
\end{tabular}




\begin{tabular}{|c|c|}
\hline $\mathrm{N} 2-\mathrm{Fe} 1-\mathrm{N} 3$ & $88.42(10)$ \\
\hline $\mathrm{N} 1{ }^{\mathrm{i}}-\mathrm{Fe} 1-\mathrm{N} 2$ & $92.68(10)$ \\
\hline $\mathrm{N} 2-\mathrm{Fe} 1-\mathrm{N} 2^{\mathrm{i}}$ & 180.00 \\
\hline $\mathrm{N} 2-\mathrm{Fe} 1-\mathrm{N} 3^{\mathrm{i}}$ & $91.58(10)$ \\
\hline $\mathrm{N} 1{ }^{\mathrm{i}}-\mathrm{Fe} 1-\mathrm{N} 3$ & $91.62(9)$ \\
\hline $\mathrm{N} 2 \mathrm{i}-\mathrm{Fe} 1-\mathrm{N} 3$ & $91.58(10)$ \\
\hline $\mathrm{N} 3-\mathrm{Fe} 1-\mathrm{N} 3^{\mathrm{i}}$ & 180.00 \\
\hline 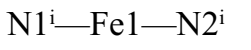 & $87.32(10)$ \\
\hline $\mathrm{N} 1^{\mathrm{i}}-\mathrm{Fe} 1-\mathrm{N} 3^{\mathrm{i}}$ & $88.38(9)$ \\
\hline $\mathrm{N} 2^{\mathrm{i}}-\mathrm{Fe} 1-\mathrm{N} 3^{\mathrm{i}}$ & $88.42(10)$ \\
\hline $\mathrm{N} 7-\mathrm{Fe} 2-\mathrm{N} 8^{\mathrm{ii}}$ & $91.93(10)$ \\
\hline $\mathrm{N} 7-\mathrm{Fe} 2-\mathrm{N} 9^{\mathrm{ii}}$ & $91.96(11)$ \\
\hline $\mathrm{N} 8-\mathrm{Fe} 2-\mathrm{N} 9$ & $87.58(10)$ \\
\hline $\mathrm{N} 7{ }^{\mathrm{ii}}-\mathrm{Fe} 2-\mathrm{N} 8$ & $91.93(10)$ \\
\hline $\mathrm{N} 8-\mathrm{Fe} 2-\mathrm{N} 8^{\mathrm{ii}}$ & 180.00 \\
\hline $\mathrm{N} 8-\mathrm{Fe} 2-\mathrm{N} 9^{\mathrm{ii}}$ & $92.42(10)$ \\
\hline $\mathrm{N} 7{ }^{\mathrm{ii}}-\mathrm{Fe} 2-\mathrm{N} 9$ & $91.96(11)$ \\
\hline $\mathrm{N} 8{ }^{\mathrm{ii}}-\mathrm{Fe} 2-\mathrm{N} 9$ & $92.42(10)$ \\
\hline $\mathrm{N} 9-\mathrm{Fe} 2-\mathrm{N} 9^{\mathrm{ii}}$ & 180.00 \\
\hline $\mathrm{N} 7^{\mathrm{ii}}-\mathrm{Fe} 2-\mathrm{N} 8^{\mathrm{ii}}$ & $88.07(10)$ \\
\hline $\mathrm{N} 7^{\mathrm{ii}}-\mathrm{Fe} 2-\mathrm{N} 9^{\mathrm{ii}}$ & $88.04(11)$ \\
\hline $\mathrm{N} 8^{\mathrm{ii}}-\mathrm{Fe} 2-\mathrm{N} 9^{\mathrm{ii}}$ & $87.58(10)$ \\
\hline $\mathrm{N} 7-\mathrm{Fe} 2-\mathrm{N} 8$ & 88.07 (10) \\
\hline $\mathrm{N} 7-\mathrm{Fe} 2-\mathrm{N} 9$ & 88.04 (11) \\
\hline $\mathrm{N} 7-\mathrm{Fe} 2-\mathrm{N} 7^{\mathrm{ii}}$ & 180.00 \\
\hline $\mathrm{Fe} 1-\mathrm{N} 1-\mathrm{N} 4$ & $120.8(2)$ \\
\hline $\mathrm{Fe} 1-\mathrm{N} 1-\mathrm{C} 1$ & $132.2(2)$ \\
\hline $\mathrm{N} 4-\mathrm{N} 1-\mathrm{C} 1$ & $106.9(2)$ \\
\hline $\mathrm{Fe} 1-\mathrm{N} 2-\mathrm{N} 5$ & $120.13(19)$ \\
\hline $\mathrm{Fe} 1-\mathrm{N} 2-\mathrm{C} 4$ & $132.79(19)$ \\
\hline $\mathrm{N} 5-\mathrm{N} 2-\mathrm{C} 4$ & $106.7(3)$ \\
\hline $\mathrm{Fe} 1-\mathrm{N} 3-\mathrm{N} 6$ & $120.79(17)$ \\
\hline $\mathrm{Fe} 1-\mathrm{N} 3-\mathrm{C} 7$ & $132.2(2)$ \\
\hline $\mathrm{N} 6-\mathrm{N} 3-\mathrm{C} 7$ & $106.9(2)$ \\
\hline $\mathrm{N} 1-\mathrm{N} 4-\mathrm{C} 3$ & $109.0(3)$ \\
\hline $\mathrm{N} 1-\mathrm{N} 4-\mathrm{B} 1$ & $118.9(2)$ \\
\hline $\mathrm{C} 3-\mathrm{N} 4-\mathrm{B} 1$ & $132.0(3)$ \\
\hline $\mathrm{N} 2-\mathrm{N} 5-\mathrm{B} 1$ & $119.0(3)$ \\
\hline $\mathrm{C} 6-\mathrm{N} 5-\mathrm{B} 1$ & $132.0(3)$ \\
\hline $\mathrm{N} 2-\mathrm{N} 5-\mathrm{C} 6$ & $109.0(3)$ \\
\hline $\mathrm{C} 9-\mathrm{N} 6-\mathrm{B} 1$ & $131.7(3)$ \\
\hline $\mathrm{N} 3-\mathrm{N} 6-\mathrm{C} 9$ & $109.5(2)$ \\
\hline $\mathrm{N} 3-\mathrm{N} 6-\mathrm{B} 1$ & $118.8(2)$ \\
\hline $\mathrm{N} 1-\mathrm{C} 1-\mathrm{C} 2$ & $110.4(3)$ \\
\hline $\mathrm{C} 1-\mathrm{C} 2-\mathrm{C} 3$ & $104.7(3)$ \\
\hline $\mathrm{N} 4-\mathrm{C} 3-\mathrm{C} 2$ & 108.9 \\
\hline $\mathrm{N} 2-\mathrm{C} 4-\mathrm{C} 5$ & $110.4(3)$ \\
\hline $\mathrm{C} 4-\mathrm{C} 5-\mathrm{C} 6$ & 105.0 \\
\hline
\end{tabular}

\begin{tabular}{|c|c|}
\hline $\mathrm{B} 1-\mathrm{C} 10-\mathrm{H} 10 \mathrm{~B}$ & 107.00 \\
\hline $\mathrm{H} 10 \mathrm{~A}-\mathrm{C} 10-\mathrm{H} 10 \mathrm{~B}$ & 107.00 \\
\hline $\mathrm{B} 1-\mathrm{C} 10-\mathrm{H} 10 \mathrm{~A}$ & 107.00 \\
\hline $\mathrm{N} 7-\mathrm{N} 10-\mathrm{C} 16$ & $109.8(2)$ \\
\hline $\mathrm{N} 7-\mathrm{N} 10-\mathrm{B} 2$ & $118.9(2)$ \\
\hline $\mathrm{C} 11-\mathrm{C} 10-\mathrm{H} 10 \mathrm{~A}$ & 107.00 \\
\hline $\mathrm{N} 8-\mathrm{N} 11-\mathrm{C} 19$ & $109.7(2)$ \\
\hline $\mathrm{N} 8-\mathrm{N} 11-\mathrm{B} 2$ & $119.3(2)$ \\
\hline $\mathrm{C} 19-\mathrm{N} 11-\mathrm{B} 2$ & $131.0(2)$ \\
\hline $\mathrm{C} 12-\mathrm{C} 11-\mathrm{H} 11 \mathrm{~A}$ & 109.00 \\
\hline $\mathrm{C} 10-\mathrm{C} 11-\mathrm{H} 11 \mathrm{~A}$ & 109.00 \\
\hline $\mathrm{C} 10-\mathrm{C} 11-\mathrm{H} 11 \mathrm{~B}$ & 109.00 \\
\hline $\mathrm{C} 12-\mathrm{C} 11-\mathrm{H} 11 \mathrm{~B}$ & 109.00 \\
\hline $\mathrm{H} 11 \mathrm{~A}-\mathrm{C} 11-\mathrm{H} 11 \mathrm{~B}$ & 108.00 \\
\hline N9-N12-C22 & $109.8(3)$ \\
\hline $\mathrm{C} 11-\mathrm{C} 12-\mathrm{H} 12 \mathrm{~A}$ & 109.00 \\
\hline $\mathrm{C} 11-\mathrm{C} 12-\mathrm{H} 12 \mathrm{~B}$ & 109.00 \\
\hline $\mathrm{C} 13-\mathrm{C} 12-\mathrm{H} 12 \mathrm{~A}$ & 109.00 \\
\hline $\mathrm{C} 13-\mathrm{C} 12-\mathrm{H} 12 \mathrm{~B}$ & 109.00 \\
\hline $\mathrm{H} 12 \mathrm{~A}-\mathrm{C} 12-\mathrm{H} 12 \mathrm{~B}$ & 108.00 \\
\hline $\mathrm{C} 22-\mathrm{N} 12-\mathrm{B} 2$ & $131.3(2)$ \\
\hline $\mathrm{N} 9-\mathrm{N} 12-\mathrm{B} 2$ & $118.9(2)$ \\
\hline $\mathrm{C} 12-\mathrm{C} 13-\mathrm{H} 13 \mathrm{~B}$ & 109.00 \\
\hline $\mathrm{C} 12-\mathrm{C} 13-\mathrm{H} 13 \mathrm{C}$ & 109.00 \\
\hline $\mathrm{H} 13 \mathrm{~A}-\mathrm{C} 13-\mathrm{H} 13 \mathrm{C}$ & 110.00 \\
\hline $\mathrm{H} 13 \mathrm{~B}-\mathrm{C} 13-\mathrm{H} 13 \mathrm{C}$ & 110.00 \\
\hline $\mathrm{H} 13 \mathrm{~A}-\mathrm{C} 13-\mathrm{H} 13 \mathrm{~B}$ & 109.00 \\
\hline $\mathrm{C} 12-\mathrm{C} 13-\mathrm{H} 13 \mathrm{~A}$ & 109.00 \\
\hline $\mathrm{N} 7-\mathrm{C} 14-\mathrm{C} 15$ & $110.6(3)$ \\
\hline $\mathrm{C} 14-\mathrm{C} 15-\mathrm{C} 16$ & $104.9(3)$ \\
\hline $\mathrm{N} 10-\mathrm{C} 16-\mathrm{C} 15$ & $108.1(3)$ \\
\hline $\mathrm{N} 8-\mathrm{C} 17-\mathrm{C} 18$ & $110.5(3)$ \\
\hline $\mathrm{C} 17-\mathrm{C} 18-\mathrm{C} 19$ & $104.8(3)$ \\
\hline $\mathrm{N} 11-\mathrm{C} 19-\mathrm{C} 18$ & $108.3(3)$ \\
\hline N9- $\mathrm{C} 20-\mathrm{C} 21$ & $111.2(3)$ \\
\hline $\mathrm{C} 20-\mathrm{C} 21-\mathrm{C} 22$ & $104.5(3)$ \\
\hline $\mathrm{N} 12-\mathrm{C} 22-\mathrm{C} 21$ & $108.6(3)$ \\
\hline $\mathrm{C} 24-\mathrm{C} 23-\mathrm{B} 2$ & $119.3(3)$ \\
\hline $\mathrm{C} 23-\mathrm{C} 24-\mathrm{C} 25$ & $112.5(3)$ \\
\hline $\mathrm{C} 24-\mathrm{C} 25-\mathrm{C} 26$ & $114.0(3)$ \\
\hline $\mathrm{N} 10-\mathrm{B} 2-\mathrm{N} 11$ & $105.5(2)$ \\
\hline $\mathrm{N} 10-\mathrm{B} 2-\mathrm{N} 12$ & $105.8(2)$ \\
\hline $\mathrm{N} 10-\mathrm{B} 2-\mathrm{C} 23$ & $111.7(2)$ \\
\hline $\mathrm{N} 11-\mathrm{B} 2-\mathrm{N} 12$ & $106.2(2)$ \\
\hline $\mathrm{N} 11-\mathrm{B} 2-\mathrm{C} 23$ & $114.2(2)$ \\
\hline $\mathrm{N} 12-\mathrm{B} 2-\mathrm{C} 23$ & $112.8(2)$ \\
\hline $\mathrm{N} 7-\mathrm{C} 14-\mathrm{H} 14$ & 125.00 \\
\hline $\mathrm{C} 15-\mathrm{C} 14-\mathrm{H} 14$ & 125.00 \\
\hline
\end{tabular}




\begin{tabular}{|c|c|c|c|}
\hline $\mathrm{N} 5-\mathrm{C} 6-\mathrm{C} 5$ & $108.9(3)$ & $\mathrm{C} 14-\mathrm{C} 15-\mathrm{H} 15$ & 128.00 \\
\hline $\mathrm{N} 3-\mathrm{C} 7-\mathrm{C} 8$ & $110.1(3)$ & $\mathrm{C} 16-\mathrm{C} 15-\mathrm{H} 15$ & 128.00 \\
\hline $\mathrm{C} 7-\mathrm{C} 8-\mathrm{C} 9$ & $104.8(3)$ & $\mathrm{N} 10-\mathrm{C} 16-\mathrm{H} 16$ & 126.00 \\
\hline $\mathrm{N} 6-\mathrm{C} 9-\mathrm{C} 8$ & $108.6(3)$ & $\mathrm{C} 15-\mathrm{C} 16-\mathrm{H} 16$ & 126.00 \\
\hline $\mathrm{C} 11-\mathrm{C} 10-\mathrm{B} 1$ & $119.7(3)$ & $\mathrm{N} 8-\mathrm{C} 17-\mathrm{H} 17$ & 125.00 \\
\hline $\mathrm{C} 10-\mathrm{C} 11-\mathrm{C} 12$ & $114.2(3)$ & $\mathrm{C} 18-\mathrm{C} 17-\mathrm{H} 17$ & 125.00 \\
\hline $\mathrm{C} 11-\mathrm{C} 12-\mathrm{C} 13$ & $114.0(3)$ & $\mathrm{C} 17-\mathrm{C} 18-\mathrm{H} 18$ & 128.00 \\
\hline $\mathrm{N} 4-\mathrm{B} 1-\mathrm{N} 5$ & $106.9(3)$ & $\mathrm{C} 19-\mathrm{C} 18-\mathrm{H} 18$ & 128.00 \\
\hline $\mathrm{N} 4-\mathrm{B} 1-\mathrm{N} 6$ & $105.1(2)$ & $\mathrm{N} 11-\mathrm{C} 19-\mathrm{H} 19$ & 126.00 \\
\hline $\mathrm{N} 4-\mathrm{B} 1-\mathrm{C} 10$ & $113.4(3)$ & $\mathrm{C} 18-\mathrm{C} 19-\mathrm{H} 19$ & 126.00 \\
\hline $\mathrm{N} 5-\mathrm{B} 1-\mathrm{N} 6$ & $105.4(2)$ & $\mathrm{N} 9-\mathrm{C} 20-\mathrm{H} 20$ & 124.00 \\
\hline $\mathrm{N} 5-\mathrm{B} 1-\mathrm{C} 10$ & $114.4(3)$ & $\mathrm{C} 21-\mathrm{C} 20-\mathrm{H} 20$ & 124.00 \\
\hline $\mathrm{N} 6-\mathrm{B} 1-\mathrm{C} 10$ & $111.0(3)$ & $\mathrm{C} 20-\mathrm{C} 21-\mathrm{H} 21$ & 128.00 \\
\hline $\mathrm{N} 1-\mathrm{C} 1-\mathrm{H} 1$ & 125.00 & $\mathrm{C} 22-\mathrm{C} 21-\mathrm{H} 21$ & 128.00 \\
\hline $\mathrm{C} 2-\mathrm{C} 1-\mathrm{H} 1$ & 125.00 & $\mathrm{~N} 12-\mathrm{C} 22-\mathrm{H} 22$ & 126.00 \\
\hline $\mathrm{C} 1-\mathrm{C} 2-\mathrm{H} 2$ & 128.00 & $\mathrm{C} 21-\mathrm{C} 22-\mathrm{H} 22$ & 126.00 \\
\hline $\mathrm{C} 3-\mathrm{C} 2-\mathrm{H} 2$ & 128.00 & $\mathrm{C} 24-\mathrm{C} 23-\mathrm{H} 23 \mathrm{~A}$ & 108.00 \\
\hline $\mathrm{N} 4-\mathrm{C} 3-\mathrm{H} 3$ & 125.00 & $\mathrm{C} 24-\mathrm{C} 23-\mathrm{H} 23 \mathrm{~B}$ & 108.00 \\
\hline $\mathrm{C} 2-\mathrm{C} 3-\mathrm{H} 3$ & 126.00 & $\mathrm{~B} 2-\mathrm{C} 23-\mathrm{H} 23 \mathrm{~A}$ & 107.00 \\
\hline $\mathrm{N} 2-\mathrm{C} 4-\mathrm{H} 4$ & 125.00 & $\mathrm{~B} 2-\mathrm{C} 23-\mathrm{H} 23 \mathrm{~B}$ & 108.00 \\
\hline $\mathrm{C} 5-\mathrm{C} 4-\mathrm{H} 4$ & 125.00 & $\mathrm{H} 23 \mathrm{~A}-\mathrm{C} 23-\mathrm{H} 23 \mathrm{~B}$ & 107.00 \\
\hline $\mathrm{C} 4-\mathrm{C} 5-\mathrm{H} 5$ & 128.00 & $\mathrm{C} 23-\mathrm{C} 24-\mathrm{H} 24 \mathrm{~A}$ & 109.00 \\
\hline $\mathrm{C} 6-\mathrm{C} 5-\mathrm{H} 5$ & 127.00 & $\mathrm{C} 23-\mathrm{C} 24-\mathrm{H} 24 \mathrm{~B}$ & 109.00 \\
\hline $\mathrm{N} 5-\mathrm{C} 6-\mathrm{H} 6$ & 126.00 & $\mathrm{C} 25-\mathrm{C} 24-\mathrm{H} 24 \mathrm{~A}$ & 109.00 \\
\hline $\mathrm{C} 5-\mathrm{C} 6-\mathrm{H} 6$ & 126.00 & $\mathrm{C} 25-\mathrm{C} 24-\mathrm{H} 24 \mathrm{~B}$ & 109.00 \\
\hline $\mathrm{Fe} 2-\mathrm{N} 7-\mathrm{N} 10$ & $120.6(2)$ & $\mathrm{H} 24 \mathrm{~A}-\mathrm{C} 24-\mathrm{H} 24 \mathrm{~B}$ & 108.00 \\
\hline $\mathrm{N} 3-\mathrm{C} 7-\mathrm{H} 7$ & 125.00 & $\mathrm{C} 24-\mathrm{C} 25-\mathrm{H} 25 \mathrm{~A}$ & 109.00 \\
\hline $\mathrm{C} 8-\mathrm{C} 7-\mathrm{H} 7$ & 125.00 & $\mathrm{C} 24-\mathrm{C} 25-\mathrm{H} 25 \mathrm{~B}$ & 109.00 \\
\hline $\mathrm{N} 10-\mathrm{N} 7-\mathrm{C} 14$ & $106.5(3)$ & $\mathrm{C} 26-\mathrm{C} 25-\mathrm{H} 25 \mathrm{~A}$ & 109.00 \\
\hline $\mathrm{Fe} 2-\mathrm{N} 7-\mathrm{C} 14$ & $132.8(2)$ & $\mathrm{C} 26-\mathrm{C} 25-\mathrm{H} 25 \mathrm{~B}$ & 109.00 \\
\hline $\mathrm{C} 9-\mathrm{C} 8-\mathrm{H} 8$ & 128.00 & $\mathrm{H} 25 \mathrm{~A}-\mathrm{C} 25-\mathrm{H} 25 \mathrm{~B}$ & 108.00 \\
\hline $\mathrm{C} 7-\mathrm{C} 8-\mathrm{H} 8$ & 128.00 & $\mathrm{C} 25-\mathrm{C} 26-\mathrm{H} 26 \mathrm{~A}$ & 110.00 \\
\hline $\mathrm{Fe} 2-\mathrm{N} 8-\mathrm{N} 11$ & $120.43(17)$ & $\mathrm{C} 25-\mathrm{C} 26-\mathrm{H} 26 \mathrm{~B}$ & 109.00 \\
\hline $\mathrm{Fe} 2-\mathrm{N} 8-\mathrm{C} 17$ & $132.88(19)$ & $\mathrm{C} 25-\mathrm{C} 26-\mathrm{H} 26 \mathrm{C}$ & 109.00 \\
\hline $\mathrm{N} 11-\mathrm{N} 8-\mathrm{C} 17$ & $106.7(2)$ & $\mathrm{H} 26 \mathrm{~A}-\mathrm{C} 26-\mathrm{H} 26 \mathrm{~B}$ & 109.00 \\
\hline $\mathrm{Fe} 2-\mathrm{N} 9-\mathrm{N} 12$ & $120.7(2)$ & $\mathrm{H} 26 \mathrm{~A}-\mathrm{C} 26-\mathrm{H} 26 \mathrm{C}$ & 109.00 \\
\hline $\mathrm{Fe} 2-\mathrm{N} 9-\mathrm{C} 20$ & $133.5(2)$ & $\mathrm{H} 26 \mathrm{~B}-\mathrm{C} 26-\mathrm{H} 26 \mathrm{C}$ & 109.00 \\
\hline $\mathrm{N} 2-\mathrm{Fe} 1-\mathrm{N} 1-\mathrm{N} 4$ & $-45.0(2)$ & $\mathrm{N} 2-\mathrm{N} 5-\mathrm{B} 1-\mathrm{N} 6$ & $60.6(3)$ \\
\hline $\mathrm{N} 2-\mathrm{Fe} 1-\mathrm{N} 1-\mathrm{C} 1$ & $138.6(3)$ & $\mathrm{C} 6-\mathrm{N} 5-\mathrm{B} 1-\mathrm{N} 6$ & $-119.3(3)$ \\
\hline $\mathrm{N} 3-\mathrm{Fe} 1-\mathrm{N} 1-\mathrm{N} 4$ & $43.5(2)$ & $\mathrm{C} 6-\mathrm{N} 5-\mathrm{B} 1-\mathrm{N} 4$ & $129.3(3)$ \\
\hline $\mathrm{N} 3-\mathrm{Fe} 1-\mathrm{N} 1-\mathrm{C} 1$ & $-132.9(3)$ & $\mathrm{C} 6-\mathrm{N} 5-\mathrm{B} 1-\mathrm{C} 10$ & $2.9(4)$ \\
\hline $\mathrm{N} 2-\mathrm{Fe} 1-\mathrm{N} 1-\mathrm{N} 4$ & $135.0(2)$ & $\mathrm{N} 2-\mathrm{N} 5-\mathrm{B} 1-\mathrm{C} 10$ & $-177.3(2)$ \\
\hline $\mathrm{N} 2-\mathrm{Fe} 1-\mathrm{N} 1-\mathrm{C} 1$ & $-41.4(3)$ & $\mathrm{C} 9-\mathrm{N} 6-\mathrm{B} 1-\mathrm{N} 5$ & $126.1(4)$ \\
\hline $\mathrm{N} 3-\mathrm{Fe} 1-\mathrm{N} 1-\mathrm{N} 4$ & $-136.5(2)$ & $\mathrm{N} 3-\mathrm{N} 6-\mathrm{B} 1-\mathrm{N} 5$ & $-54.5(3)$ \\
\hline $\mathrm{N} 3-\mathrm{Fe} 1-\mathrm{N} 1-\mathrm{C} 1$ & $47.1(3)$ & $\mathrm{N} 3-\mathrm{N} 6-\mathrm{B} 1-\mathrm{N} 4$ & $58.3(3)$ \\
\hline $\mathrm{N} 1-\mathrm{Fe} 1-\mathrm{N} 2-\mathrm{N} 5$ & $48.8(2)$ & $\mathrm{C} 9-\mathrm{N} 6-\mathrm{B} 1-\mathrm{N} 4$ & $-121.2(4)$ \\
\hline $\mathrm{N} 1-\mathrm{Fe} 1-\mathrm{N} 2-\mathrm{C} 4$ & $-138.9(3)$ & $\mathrm{B} 1-\mathrm{N} 6-\mathrm{C} 9-\mathrm{C} 8$ & $179.9(3)$ \\
\hline
\end{tabular}




\begin{tabular}{|c|c|c|c|}
\hline $\mathrm{N} 3-\mathrm{Fe} 1-\mathrm{N} 2-\mathrm{N} 5$ & $-39.7(2)$ & $\mathrm{N} 3-\mathrm{N} 6-\mathrm{B} 1-\mathrm{C} 10$ & $-178.8(3)$ \\
\hline $\mathrm{N} 3-\mathrm{Fe} 1-\mathrm{N} 2-\mathrm{C} 4$ & $132.7(3)$ & $\mathrm{C} 9-\mathrm{N} 6-\mathrm{B} 1-\mathrm{C} 10$ & $1.8(5)$ \\
\hline $\mathrm{N} 1{ }^{\mathrm{i}}-\mathrm{Fe} 1-\mathrm{N} 2-\mathrm{N} 5$ & $-131.2(2)$ & $\mathrm{N} 3-\mathrm{N} 6-\mathrm{C} 9-\mathrm{C} 8$ & $0.5(4)$ \\
\hline $\mathrm{N} 1-\mathrm{Fe} 1-\mathrm{N} 2-\mathrm{C} 4$ & $41.1(3)$ & $\mathrm{N} 1-\mathrm{C} 1-\mathrm{C} 2-\mathrm{C} 3$ & $0.1(4)$ \\
\hline 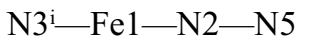 & $140.3(2)$ & $\mathrm{C} 1-\mathrm{C} 2-\mathrm{C} 3-\mathrm{N} 4$ & $0.0(3)$ \\
\hline 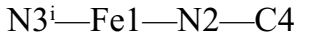 & $-47.3(3)$ & $\mathrm{N} 2-\mathrm{C} 4-\mathrm{C} 5-\mathrm{C} 6$ & $0.0(4)$ \\
\hline $\mathrm{N} 1-\mathrm{Fe} 1-\mathrm{N} 3-\mathrm{N} 6$ & $-42.0(2)$ & $\mathrm{C} 4-\mathrm{C} 5-\mathrm{C} 6-\mathrm{N} 5$ & $-0.7(4)$ \\
\hline $\mathrm{N} 1-\mathrm{Fe} 1-\mathrm{N} 3-\mathrm{C} 7$ & $133.5(3)$ & $\mathrm{N} 3-\mathrm{C} 7-\mathrm{C} 8-\mathrm{C} 9$ & $0.2(4)$ \\
\hline $\mathrm{N} 2-\mathrm{Fe} 1-\mathrm{N} 3-\mathrm{N} 6$ & $45.4(2)$ & $\mathrm{C} 7-\mathrm{C} 8-\mathrm{C} 9-\mathrm{N} 6$ & $-0.4(4)$ \\
\hline $\mathrm{N} 2-\mathrm{Fe} 1-\mathrm{N} 3-\mathrm{C} 7$ & $-139.1(3)$ & $\mathrm{B} 1-\mathrm{C} 10-\mathrm{C} 11-\mathrm{C} 12$ & $173.6(3)$ \\
\hline $\mathrm{N} 1-\mathrm{Fe} 1-\mathrm{N} 3-\mathrm{N} 6$ & $138.0(2)$ & $\mathrm{C} 11-\mathrm{C} 10-\mathrm{B} 1-\mathrm{N} 5$ & $60.5(4)$ \\
\hline $\mathrm{N} 1{ }^{\mathrm{i}}-\mathrm{Fe} 1-\mathrm{N} 3-\mathrm{C} 7$ & $-46.5(3)$ & $\mathrm{C} 11-\mathrm{C} 10-\mathrm{B} 1-\mathrm{N} 6$ & $179.6(3)$ \\
\hline $\mathrm{N} 2-\mathrm{Fe} 1-\mathrm{N} 3-\mathrm{N} 6$ & $-134.6(2)$ & $\mathrm{C} 11-\mathrm{C} 10-\mathrm{B} 1-\mathrm{N} 4$ & $-62.5(4)$ \\
\hline $\mathrm{N} 22^{\mathrm{i}}-\mathrm{Fe} 1-\mathrm{N} 3-\mathrm{C} 7$ & $40.9(3)$ & $\mathrm{C} 10-\mathrm{C} 11-\mathrm{C} 12-\mathrm{C} 13$ & $67.1(4)$ \\
\hline $\mathrm{N} 9-\mathrm{Fe} 2-\mathrm{N} 8-\mathrm{N} 11$ & $-44.3(2)$ & $\mathrm{Fe} 2-\mathrm{N} 7-\mathrm{N} 10-\mathrm{C} 16$ & $175.6(2)$ \\
\hline $\mathrm{N} 9-\mathrm{Fe} 2-\mathrm{N} 8-\mathrm{C} 17$ & $135.2(3)$ & $\mathrm{Fe} 2-\mathrm{N} 7-\mathrm{N} 10-\mathrm{B} 2$ & $-2.7(3)$ \\
\hline $\mathrm{N} 7{ }^{\mathrm{ii}}-\mathrm{Fe} 2-\mathrm{N} 8-\mathrm{N} 11$ & $-136.2(2)$ & $\mathrm{C} 14-\mathrm{N} 7-\mathrm{N} 10-\mathrm{C} 16$ & $-0.9(3)$ \\
\hline $\mathrm{N} 7{ }^{\mathrm{ii}}-\mathrm{Fe} 2-\mathrm{N} 8-\mathrm{C} 17$ & $43.3(3)$ & $\mathrm{C} 14-\mathrm{N} 7-\mathrm{N} 10-\mathrm{B} 2$ & $-179.3(3)$ \\
\hline $\mathrm{N} 9{ }^{\mathrm{ii}}-\mathrm{Fe} 2-\mathrm{N} 8-\mathrm{N} 11$ & $135.7(2)$ & $\mathrm{Fe} 2-\mathrm{N} 7-\mathrm{C} 14-\mathrm{C} 15$ & $-175.1(2)$ \\
\hline $\mathrm{N} 9^{\mathrm{ii}}-\mathrm{Fe} 2-\mathrm{N} 8-\mathrm{C} 17$ & $-44.8(3)$ & $\mathrm{N} 10-\mathrm{N} 7-\mathrm{C} 14-\mathrm{C} 15$ & $0.8(4)$ \\
\hline $\mathrm{N} 7-\mathrm{Fe} 2-\mathrm{N} 9-\mathrm{N} 12$ & $-42.2(2)$ & $\mathrm{Fe} 2-\mathrm{N} 8-\mathrm{N} 11-\mathrm{C} 19$ & $-179.9(2)$ \\
\hline $\mathrm{N} 7-\mathrm{Fe} 2-\mathrm{N} 9-\mathrm{C} 20$ & $136.2(3)$ & $\mathrm{Fe} 2-\mathrm{N} 8-\mathrm{N} 11-\mathrm{B} 2$ & $-0.3(4)$ \\
\hline $\mathrm{N} 8-\mathrm{Fe} 2-\mathrm{N} 9-\mathrm{N} 12$ & $46.0(2)$ & $\mathrm{C} 17-\mathrm{N} 8-\mathrm{N} 11-\mathrm{C} 19$ & $0.5(4)$ \\
\hline $\mathrm{N} 8-\mathrm{Fe} 2-\mathrm{N} 9-\mathrm{C} 20$ & $-135.7(3)$ & $\mathrm{C} 17-\mathrm{N} 8-\mathrm{N} 11-\mathrm{B} 2$ & $-179.9(3)$ \\
\hline $\mathrm{N} 7{ }^{\mathrm{ii}}-\mathrm{Fe} 2-\mathrm{N} 9-\mathrm{N} 12$ & $137.8(2)$ & $\mathrm{Fe} 2-\mathrm{N} 8-\mathrm{C} 17-\mathrm{C} 18$ & $-179.7(2)$ \\
\hline $\mathrm{N} 7{ }^{\mathrm{ii}}-\mathrm{Fe} 2-\mathrm{N} 9-\mathrm{C} 20$ & $-43.8(3)$ & $\mathrm{N} 11-\mathrm{N} 8-\mathrm{C} 17-\mathrm{C} 18$ & $-0.2(4)$ \\
\hline $\mathrm{N} 8{ }^{\mathrm{ii}}-\mathrm{Fe} 2-\mathrm{N} 9-\mathrm{N} 12$ & $-134.1(2)$ & $\mathrm{Fe} 2-\mathrm{N} 9-\mathrm{N} 12-\mathrm{C} 22$ & $178.28(19)$ \\
\hline $\mathrm{N} 8 \mathrm{ii}-\mathrm{Fe} 2-\mathrm{N} 9-\mathrm{C} 20$ & $44.3(3)$ & $\mathrm{Fe} 2-\mathrm{N} 9-\mathrm{N} 12-\mathrm{B} 2$ & $-3.0(3)$ \\
\hline $\mathrm{N} 9$ ii-Fe2-N7-C14 & $41.0(3)$ & $\mathrm{C} 20-\mathrm{N} 9-\mathrm{N} 12-\mathrm{C} 22$ & $-0.5(3)$ \\
\hline $\mathrm{N} 7-\mathrm{Fe} 2-\mathrm{N} 8-\mathrm{N} 11$ & $43.8(2)$ & $\mathrm{C} 20-\mathrm{N} 9-\mathrm{N} 12-\mathrm{B} 2$ & $178.2(2)$ \\
\hline $\mathrm{N} 7-\mathrm{Fe} 2-\mathrm{N} 8-\mathrm{C} 17$ & $-136.7(3)$ & $\mathrm{Fe} 2-\mathrm{N} 9-\mathrm{C} 20-\mathrm{C} 21$ & $-178.0(2)$ \\
\hline $\mathrm{N} 9-\mathrm{Fe} 2-\mathrm{N} 7-\mathrm{N} 10$ & $45.5(2)$ & $\mathrm{N} 12-\mathrm{N} 9-\mathrm{C} 20-\mathrm{C} 21$ & $0.6(3)$ \\
\hline N9-Fe2-N7-C14 & $-139.0(3)$ & $\mathrm{N} 7-\mathrm{N} 10-\mathrm{C} 16-\mathrm{C} 15$ & $0.7(4)$ \\
\hline $\mathrm{N} 8{ }^{\mathrm{ii}}-\mathrm{Fe} 2-\mathrm{N} 7-\mathrm{N} 10$ & $137.8(2)$ & $\mathrm{B} 2-\mathrm{N} 10-\mathrm{C} 16-\mathrm{C} 15$ & $178.7(3)$ \\
\hline $\mathrm{N} 8{ }^{\mathrm{ii}}-\mathrm{Fe} 2-\mathrm{N} 7-\mathrm{C} 14$ & $-46.7(3)$ & $\mathrm{C} 16-\mathrm{N} 10-\mathrm{B} 2-\mathrm{N} 12$ & $127.7(3)$ \\
\hline $\mathrm{N} 9 \mathrm{ii}-\mathrm{Fe} 2-\mathrm{N} 7-\mathrm{N} 10$ & $-134.5(2)$ & $\mathrm{N} 7-\mathrm{N} 10-\mathrm{B} 2-\mathrm{C} 23$ & $-177.5(3)$ \\
\hline $\mathrm{N} 8-\mathrm{Fe} 2-\mathrm{N} 7-\mathrm{N} 10$ & $-42.2(2)$ & $\mathrm{C} 16-\mathrm{N} 10-\mathrm{B} 2-\mathrm{C} 23$ & $4.6(4)$ \\
\hline $\mathrm{N} 8-\mathrm{Fe} 2-\mathrm{N} 7-\mathrm{C} 14$ & $133.3(3)$ & $\mathrm{C} 16-\mathrm{N} 10-\mathrm{B} 2-\mathrm{N} 11$ & $-120.0(3)$ \\
\hline $\mathrm{Fe} 1-\mathrm{N} 1-\mathrm{N} 4-\mathrm{C} 3$ & $-177.10(19)$ & $\mathrm{N} 7-\mathrm{N} 10-\mathrm{B} 2-\mathrm{N} 12$ & $-54.3(3)$ \\
\hline $\mathrm{Fe} 1-\mathrm{N} 1-\mathrm{N} 4-\mathrm{B} 1$ & $0.0(3)$ & $\mathrm{N} 7-\mathrm{N} 10-\mathrm{B} 2-\mathrm{N} 11$ & $57.9(3)$ \\
\hline $\mathrm{C} 1-\mathrm{N} 1-\mathrm{N} 4-\mathrm{C} 3$ & $0.1(3)$ & $\mathrm{N} 8-\mathrm{N} 11-\mathrm{B} 2-\mathrm{N} 10$ & $-56.3(3)$ \\
\hline $\mathrm{C} 1-\mathrm{N} 1-\mathrm{N} 4-\mathrm{B} 1$ & $177.3(2)$ & $\mathrm{C} 19-\mathrm{N} 11-\mathrm{B} 2-\mathrm{N} 10$ & $123.2(3)$ \\
\hline $\mathrm{Fe} 1-\mathrm{N} 1-\mathrm{C} 1-\mathrm{C} 2$ & $176.6(2)$ & $\mathrm{C} 19-\mathrm{N} 11-\mathrm{B} 2-\mathrm{C} 23$ & $0.3(5)$ \\
\hline $\mathrm{N} 4-\mathrm{N} 1-\mathrm{C} 1-\mathrm{C} 2$ & $-0.1(3)$ & $\mathrm{B} 2-\mathrm{N} 11-\mathrm{C} 19-\mathrm{C} 18$ & $179.8(3)$ \\
\hline $\mathrm{N} 5-\mathrm{N} 2-\mathrm{C} 4-\mathrm{C} 5$ & $0.7(3)$ & $\mathrm{C} 19-\mathrm{N} 11-\mathrm{B} 2-\mathrm{N} 12$ & $-124.7(3)$ \\
\hline $\mathrm{Fe} 1-\mathrm{N} 2-\mathrm{N} 5-\mathrm{C} 6$ & $173.0(2)$ & $\mathrm{N} 8-\mathrm{N} 11-\mathrm{B} 2-\mathrm{C} 23$ & $-179.3(3)$ \\
\hline $\mathrm{Fe} 1-\mathrm{N} 2-\mathrm{N} 5-\mathrm{B} 1$ & $-6.9(3)$ & $\mathrm{N} 8-\mathrm{N} 11-\mathrm{B} 2-\mathrm{N} 12$ & $55.7(3)$ \\
\hline $\mathrm{C} 4-\mathrm{N} 2-\mathrm{N} 5-\mathrm{C} 6$ & $-1.2(3)$ & $\mathrm{N} 8-\mathrm{N} 11-\mathrm{C} 19-\mathrm{C} 18$ & $-0.6(4)$ \\
\hline
\end{tabular}




$\begin{array}{llll}\mathrm{C} 4-\mathrm{N} 2-\mathrm{N} 5-\mathrm{B} 1 & 178.9(3) & \mathrm{C} 22-\mathrm{N} 12-\mathrm{B} 2-\mathrm{N} 10 & -123.8(3) \\ \mathrm{Fe} 1-\mathrm{N} 2-\mathrm{C} 4-\mathrm{C} 5 & -172.4(2) & \mathrm{N} 9-\mathrm{N} 12-\mathrm{B} 2-\mathrm{N} 11 & -53.9(3) \\ \mathrm{C} 7-\mathrm{N} 3-\mathrm{N} 6-\mathrm{C} 9 & -0.4(4) & \mathrm{N} 9-\mathrm{N} 12-\mathrm{B} 2-\mathrm{N} 10 & 57.9(3) \\ \mathrm{C} 7-\mathrm{N} 3-\mathrm{N} 6-\mathrm{B} 1 & -179.9(3) & \mathrm{N} 9-\mathrm{N} 12-\mathrm{C} 22-\mathrm{C} 21 & 0.3(3) \\ \mathrm{Fe} 1-\mathrm{N} 3-\mathrm{C} 7-\mathrm{C} 8 & -175.8(2) & \mathrm{B} 2-\mathrm{N} 12-\mathrm{C} 22-\mathrm{C} 21 & -178.2(3) \\ \mathrm{N} 6-\mathrm{N} 3-\mathrm{C} 7-\mathrm{C} 8 & 0.1(4) & \mathrm{C} 22-\mathrm{N} 12-\mathrm{B} 2-\mathrm{C} 23 & -1.4(4) \\ \mathrm{Fe} 1-\mathrm{N} 3-\mathrm{N} 6-\mathrm{C} 9 & 176.2(2) & \mathrm{C} 22-\mathrm{N} 12-\mathrm{B} 2-\mathrm{N} 11 & 124.5(3) \\ \mathrm{Fe} 1-\mathrm{N} 3-\mathrm{N} 6-\mathrm{B} 1 & -3.4(4) & \mathrm{N} 9-\mathrm{N} 12-\mathrm{B} 2-\mathrm{C} 23 & -179.7(2) \\ \mathrm{N} 1-\mathrm{N} 4-\mathrm{C} 3-\mathrm{C} 2 & -0.1(3) & \mathrm{N} 7-\mathrm{C} 14-\mathrm{C} 15-\mathrm{C} 16 & -0.4(4) \\ \mathrm{C} 3-\mathrm{N} 4-\mathrm{B} 1-\mathrm{N} 6 & 119.8(3) & \mathrm{C} 14-\mathrm{C} 15-\mathrm{C} 16-\mathrm{N} 10 & -0.2(4) \\ \mathrm{B} 1-\mathrm{N} 4-\mathrm{C} 3-\mathrm{C} 2 & -176.7(3) & \mathrm{N} 8-\mathrm{C} 17-\mathrm{C} 18-\mathrm{C} 19 & -0.2(4) \\ \mathrm{N} 1-\mathrm{N} 4-\mathrm{B} 1-\mathrm{N} 6 & -56.6(3) & \mathrm{C} 17-\mathrm{C} 18-\mathrm{C} 19-\mathrm{N} 11 & 0.5(4) \\ \mathrm{C} 3-\mathrm{N} 4-\mathrm{B} 1-\mathrm{C} 10 & -1.5(4) & \mathrm{N} 9-\mathrm{C} 20-\mathrm{C} 21-\mathrm{C} 22 & -0.4(4) \\ \mathrm{N} 1-\mathrm{N} 4-\mathrm{B} 1-\mathrm{N} 5 & 55.1(3) & \mathrm{C} 20-\mathrm{C} 21-\mathrm{C} 22-\mathrm{N} 12 & 0.1(3) \\ \mathrm{C} 3-\mathrm{N} 4-\mathrm{B} 1-\mathrm{N} 5 & -128.5(3) & \mathrm{B} 2-\mathrm{C} 23-\mathrm{C} 24-\mathrm{C} 25 & -144.3(3) \\ \mathrm{N} 1-\mathrm{N} 4-\mathrm{B} 1-\mathrm{C} 10 & -177.9(2) & \mathrm{C} 24-\mathrm{C} 23-\mathrm{B} 2-\mathrm{N} 12 & 61.3(4) \\ \mathrm{N} 2-\mathrm{N} 5-\mathrm{B} 1-\mathrm{N} 4 & -50.9(3) & \mathrm{C} 24-\mathrm{C} 23-\mathrm{B} 2-\mathrm{N} 10 & -179.6(3) \\ \mathrm{N} 2-\mathrm{N} 5-\mathrm{C} 6-\mathrm{C} 5 & 1.2(3) & \mathrm{C} 24-\mathrm{C} 23-\mathrm{B} 2-\mathrm{N} 11 & -60.1(4) \\ \mathrm{B} 1-\mathrm{N} 5-\mathrm{C} 6-\mathrm{C} 5 & -178.9(3) & \mathrm{C} 23-\mathrm{C} 24-\mathrm{C} 25-\mathrm{C} 26 & 178.9(3)\end{array}$

Symmetry codes: (i) $-x,-y,-z+1$; (ii) $-x+1,-y,-z+1$.

Hydrogen-bond geometry $\left(A,{ }^{\circ}\right)$

$C g 2$ and $C g 11$ are the centroids of rings N2/N5/C4-C6 and N8/N11/C17-C19, respectively.

\begin{tabular}{lllll}
\hline$D-\mathrm{H} \cdots A$ & $D-\mathrm{H}$ & $\mathrm{H} \cdots A$ & $D \cdots A$ & $D-\mathrm{H} \cdots A$ \\
\hline $\mathrm{C} 5-\mathrm{H} 5 \cdots C g 11$ & 0.93 & 3.00 & $3.674(4)$ & 131 \\
$\mathrm{C} 12-\mathrm{H} 12 A \cdots C g 2^{\mathrm{iii}}$ & 0.97 & 2.87 & $3.703(3)$ & 145 \\
$\mathrm{C} 26-\mathrm{H} 26 C \cdots C g 11^{\mathrm{iv}}$ & 0.96 & 2.84 & $3.720(4)$ & 153 \\
\hline
\end{tabular}

Symmetry codes: (iii) $-x, y+1 / 2,-z+1 / 2$; (iv) $-x+1, y+1 / 2,-z+1 / 2$. 